Claremont Colleges

Scholarship@ Claremont

All HMC Faculty Publications and Research

HMC Faculty Scholarship

$12-1-2007$

\title{
Gravity-driven Thin Liquid Films with Insoluble Surfactant: Smooth Traveling Waves
}

\author{
Rachel Levy \\ Harvey Mudd College \\ Michael Shearer \\ North Carolina State University at Raleigh \\ Thomas P. Witelski \\ Duke University
}

\section{Recommended Citation}

Levy, R, Shearer, M, Witelski, TP. Gravity-driven thin liquid films with insoluble surfactant: smooth traveling waves. Eur J App Math. 2007;18(6): 679-708.

This Article is brought to you for free and open access by the HMC Faculty Scholarship at Scholarship @ Claremont. It has been accepted for inclusion in All HMC Faculty Publications and Research by an authorized administrator of Scholarship @ Claremont. For more information, please contact scholarship@cuc.claremont.edu. 


\title{
Gravity-driven thin liquid films with insoluble surfactant: smooth traveling waves
}

\author{
RACHEL LEVY ${ }^{1,2}$, MICHAEL SHEARER ${ }^{1,3}$ and THOMAS P. WITELSKI ${ }^{1}$ \\ ${ }^{1}$ Department of Mathematics, Duke University, Durham NC 27708, USA \\ ${ }^{2}$ Department of Mathematics, Harvey Mudd College, Claremont, CA 91711, USA \\ ${ }^{3}$ Department of Mathematics and Center for Research in Scientific Computation, \\ N.C. State University Raleigh, NC 27695, USA
}

(Received 29 October 2007; revised 30 October 2007)

\begin{abstract}
The flow of a thin layer of fluid down an inclined plane is modified by the presence of insoluble surfactant. For any finite surfactant mass, traveling waves are constructed for a system of lubrication equations describing the evolution of the free-surface fluid height and the surfactant concentration. The one-parameter family of solutions is investigated using perturbation theory with three small parameters: the coefficient of surface tension, the surfactant diffusivity, and the coefficient of the gravity-driven diffusive spreading of the fluid. When all three parameters are zero, the nonlinear PDE system is hyperbolic/degenerateparabolic, and admits traveling wave solutions in which the free-surface height is piecewise constant, and the surfactant concentration is piecewise linear and continuous. The jumps and corners in the traveling waves are regularized when the small parameters are nonzero; their structure is revealed through a combination of analysis and numerical simulation.
\end{abstract}

\section{Introduction}

Coating flows and their applications in physics, engineering, and biology have been the subject of decades of research, see $[22,23]$ and references therein. The mathematical study of these flows of thin liquid films on solid substrates begins with the lubrication approximation of the Stokes equations. The simplest lubrication model of gravity-driven flow of a viscous fluid down an inclined plane is the one-dimensional equation [9]

$$
h_{t}+\left(\frac{1}{3} h^{3}\right)_{x}=0
$$

in which $h(x, t)$ is the height of the film at time $t$ and $x$ is measured down the plane. A more sophisticated model $[3,19,21]$ including the additional regularizing effects of gravity-driven spreading and surface tension is

$$
h_{t}+\left(\frac{1}{3} h^{3}\right)_{x}=\beta\left(\frac{1}{3} h^{3} h_{x}\right)_{x}-\kappa\left(\frac{1}{3} h^{3} h_{x x x}\right)_{x}
$$

These equations are written in dimensionless form; the positive parameters $\beta$ and $\kappa$ are given in terms of characteristic length scale $L$, characteristic film thickness $H$, density $\rho$, 
and slope $\tan \theta$ of the inclined plane,

$$
\beta=\epsilon \cot \theta, \quad \kappa=\epsilon \gamma_{0} /\left(L^{2} \rho g \cos \theta\right), \quad \epsilon=H / L \ll 1,
$$

in which $\gamma_{0}$ is surface tension, taken to be constant, and $g$ is the gravitational constant. In this scaling, the time scale $T$ is proportional to viscosity $\mu: T=\epsilon^{-2} \mu /(L \rho g \sin \theta)$.

In this article we consider the influence of a driving force in addition to gravity, provided by the introduction of surfactants $[6,7,11,10,19,27]$. Surfactants are surface active agents that lower the surface tension of a liquid, creating spatial variations in surface tension that induce a tangential surface stress known as a Marangoni force [16]. Such forces have also been created in experiments by exploiting the dependence of surface tension on temperature $[4,5,20]$.

The equations modeling thin film flow driven by gravity and the surfactant-induced Marangoni force are, in dimensionless form,

$$
\begin{aligned}
h_{t}+\left(\frac{1}{3} h^{3}\right)_{x}-\left(\frac{1}{2} h^{2} \Gamma_{x}\right)_{x} & =\beta\left(\frac{1}{3} h^{3} h_{x}\right)_{x}-\kappa\left(\frac{1}{3} h^{3} h_{x x x}\right)_{x} \\
\Gamma_{t}+\left(\frac{1}{2} h^{2} \Gamma\right)_{x}-\left(h \Gamma \Gamma_{x}\right)_{x} & =\beta\left(\frac{1}{2} h^{2} \Gamma h_{x}\right)_{x}-\kappa\left(\frac{1}{2} h^{2} \Gamma h_{x x x}\right)_{x}+\delta \Gamma_{x x} .
\end{aligned}
$$

Here, $\Gamma(x, t)$ is the concentration of insoluble surfactant on the free surface. The coefficient $\delta=\mu D /\left(\epsilon^{2} \rho g L^{3} \sin \theta\right)$ is proportional to the inverse of the Peclet number, modeling the diffusion of surfactant molecules on the surface of the film, with diffusion constant $D$ [27]. The parameters $\beta, \kappa$ are given by (1.3), where now $\gamma_{0}$ is the maximum surface tension, occurring when the surfactant concentration is zero. In dimensional terms, we are scaling the surfactant concentration relative to some fixed concentration that is assumed to be low in comparison to the maximal value, the critical micelle concentration $\Gamma_{\mathrm{CMC}}$, i.e., $0 \leqslant \Gamma \ll \Gamma_{\mathrm{CMC}}$. We take the dimensional relation between surface tension and surfactant concentration to be linear for small $\Gamma: \gamma=\gamma_{0}-a \Gamma$ with positive constant $a$. These scalings are slightly different from those used in [6], as we have normalized the coefficient of the convective terms due to gravity (the second terms in (1.4)) to be one. Note that when $\Gamma \equiv 0$, the system reduces to the single equation (1.2).

System (1.4) is derived by applying the lubrication approximation to the twodimensional Stokes equations, as explained in [11]. Integration of the resulting equations and nondimensionalization lead to an expression for the fluid velocity $u(z)$ parallel to the inclined plane:

$$
u(z)=-\frac{1}{2}\left(1-\beta h_{x}+\kappa h_{x x x}\right)\left(z^{2}-2 h z\right)-\Gamma_{x} z \text { for } 0 \leqslant z \leqslant h .
$$

Making use of the depth-averaged velocity, the lubrication equation for the conservation of fluid mass,

$$
h_{t}+(h \bar{u})_{x}=0, \quad \bar{u}=\frac{1}{h} \int_{0}^{h} u(z) d z,
$$

yields equation (1.4a). Transport of insoluble surfactant on the free surface of the fluid is given by an advection-diffusion equation in terms of the surface velocity,

$$
\Gamma_{t}+\left(\Gamma u_{h}\right)_{x}=\delta \Gamma_{x x}, \quad u_{h}=u(h),
$$


to yield $(1.4 b)$. A detailed derivation of the equations, including details of the nondimensionalization, is described in [6]. In the case of a soluble surfactant, transport and diffusion of surfactant between the free surface and the bulk requires additional equations, presented in [17, 30], for flow on a horizontal substrate.

In this paper, we investigate the effect of the coefficients $\beta, \kappa$, and $\delta$ that control smoothing or regularizing terms in the system (1.4). The parameters $\beta$ and $\kappa$ control smoothing of $h$ in the height equation (1.4a). While $\beta$ and $\kappa$ also appear in the surfactant concentration equation $(1.4 b)$, their effect there is less clear, as they do not directly smooth $\Gamma$. Equation (1.4b) includes two terms describing the diffusion of the surfactant. On the left-hand side, the degenerate diffusion term $\left(h \Gamma \Gamma_{x}\right)_{x}$ comes from the transport of surfactant by the surface velocity $u_{h}$. On the right-hand side, the linear term $\delta \Gamma_{x x}$ models diffusion of surfactant molecules on the free surface; the dimensionless parameter $\delta$ is independent of the fluid flow, and is proportional to the diffusion constant. For $\delta=0$, the degenerate diffusion equation would yield compactly supported solutions having a $\Gamma=0$ interface that propagates at finite speed $[15,25]$. The role of the additional diffusion given by $\delta$ in regularizing $\Gamma$ and in shock formation has raised questions in previous work [24] that we explore here.

In Section 1.1 we present the ODE system describing the class of traveling wave solutions that we consider. In [14], a family of traveling waves was found for the unregularized system with $\beta=\delta=\kappa=0$. These solutions, presented in Section 2, are the starting point for our analysis of the regularized equations. In Section 3, we study regularization by second-order terms only, i.e., with $\kappa=0, \beta>0$, and $\delta \geqslant 0$. We prove the existence of a one-parameter family of traveling waves, parametrized by $\Gamma_{\max }=\max _{x} \Gamma$. We also study the structure of these waves with asymptotics and numerical simulations in the limits $\Gamma_{\max } \rightarrow \infty$ (Section 3.2), $\beta \rightarrow 0, \delta>0$ (Section 3.3), $\delta \rightarrow 0, \beta \geqslant 0$ (Section 3.4). Finally, in Section 4, we consider the role of capillarity, for which $\kappa>0$.

\subsection{Traveling wave solutions}

We seek traveling wave solutions with speed $s$,

$$
h(x, t)=h(\eta), \quad \Gamma(x, t)=\Gamma(\eta), \quad \eta=x-s t .
$$

The solution profiles satisfy the system of ODEs

$$
\begin{aligned}
-s h+\frac{1}{3} h^{3}-\frac{1}{2} h^{2} \frac{d \Gamma}{d \eta} & =\frac{1}{3} \beta h^{3} \frac{d h}{d \eta}-\frac{1}{3} \kappa h^{3} \frac{d^{3} h}{d \eta^{3}}-k_{h}, \\
-s \Gamma+\frac{1}{2} h^{2} \Gamma-h \Gamma \frac{d \Gamma}{d \eta} & =\frac{1}{2} \beta h^{2} \Gamma \frac{d h}{d \eta}+\delta \frac{d \Gamma}{d \eta}-\frac{1}{2} \kappa h^{2} \Gamma \frac{d^{3} h}{d \eta^{3}}-k_{\Gamma},
\end{aligned}
$$

in which $k_{h}, k_{\Gamma}$ are constants of integration, determined by boundary conditions, which we take in the form

$$
\begin{array}{lll}
\eta \rightarrow-\infty: & h(\eta)=h_{L}, & \Gamma(\eta)=0 \\
\eta \rightarrow \infty: & h(\eta)=h_{R}, & \Gamma(\eta)=0 .
\end{array}
$$


From boundary conditions $(1.9 c)$ and assuming that $\Gamma^{\prime}(|\eta| \rightarrow \infty) \rightarrow 0$, we deduce that $k_{\Gamma}=0$. Similarly, the speed $s$, and the other constant of integration $k_{h}$ are given in terms of upstream and downstream film heights $h_{L}, h_{R}$ :

$$
s=\frac{1}{3}\left(h_{L}^{2}+h_{L} h_{R}+h_{R}^{2}\right)>0, \quad k_{h}=\frac{1}{3} h_{L} h_{R}\left(h_{L}+h_{R}\right)>0 .
$$

These constants are independent of higher order regularizing terms occuring on the right sides of $(1.9 a b)$, hence these values apply throughout the paper. Note that the wavespeed $s$ is independent of whether surfactant is present or not; it is solely determined by the influence of gravity, and is the speed of a shock (or hydraulic jump) from $h_{L}$ to $h_{R}$ in the absence of surfactant, as in equation (1.1).

\section{Traveling waves with no regularization}

We begin our analysis of traveling waves by considering the PDE system with no regularization. Setting $\beta=\kappa=\delta=0$ reduces system (1.4) to the simpler system studied in [14]:

$$
\begin{aligned}
& h_{t}+\left(\frac{1}{3} h^{3}\right)_{x}-\left(\frac{1}{2} h^{2} \Gamma_{x}\right)_{x}=0 \\
& \Gamma_{t}+\left(\frac{1}{2} h^{2} \Gamma\right)_{x}-\left(h \Gamma \Gamma_{x}\right)_{x}=0
\end{aligned}
$$

Equation $(2.1 b)$ admits the trivial solution, $\Gamma \equiv 0$ (i.e. no surfactant present), in which case, equation $(2.1 a)$ reduces to the scalar conservation law for gravity-driven flow (1.1).

The traveling wave equations $(1.9 a b)$ with $\beta=\kappa=\delta=0$ reduce to the system

$$
-s h+\frac{1}{3} h^{3}-\frac{1}{2} h^{2} \frac{d \Gamma}{d \eta}=-k_{h}, \quad-s \Gamma+\frac{1}{2} h^{2} \Gamma-h \Gamma \frac{d \Gamma}{d \eta}=0 .
$$

For $\Gamma \equiv 0,(2.2)_{2}$ is eliminated and $(2.2)_{1}$ reduces to the polynomial $-s h+\frac{1}{3} h^{3}=-k_{h}$ which has two positive roots, $h=h_{L}$ and $h=h_{R}$, corresponding to the imposed boundary values. For nontrivial $\Gamma$, equation $(2.2)_{2}$ reduces to $-s+\frac{1}{2} h^{2}-h \Gamma^{\prime}=0$; eliminating $\Gamma^{\prime}$ between the two equations in (2.2) yields a different algebraic condition on values of $h$ :

$$
6 s h-h^{3}=12 k_{h}
$$

This polynomial equation also has two positive real roots, $h=h_{1}$ and $h=h_{2}$, with $h_{1}>h_{2}$. These roots both approach $h_{L}$ as $h_{R}$ approaches $q_{\text {crit }} h_{L}$ from below, and are complex for $h_{R}>q_{\text {crit }} h_{L}$. Here, $q_{\text {crit }}$ is the critical value of the ratio $q=h_{R} / h_{L}, q_{\text {crit }}=\frac{1}{2}(\sqrt{3}-1)$, as identified in [14]. Corresponding values for the surfactant slopes, $G \equiv \Gamma^{\prime}$, can be given in terms of heights $h_{1}, h_{2}$ and $s$ by

$$
G_{1}=\frac{h_{1}^{2}-2 s}{2 h_{1}}>0, \quad G_{2}=\frac{h_{2}^{2}-2 s}{2 h_{2}}<0,
$$

and are thus implicitly determined by upstream and downstream heights $h_{L}, h_{R}$. These formulae depend on jump conditions for $h, \Gamma^{\prime}$, as explained in detail in [14]; these are not the only solutions of the jump conditions, but are the only ones relevant for the traveling waves of this paper. 

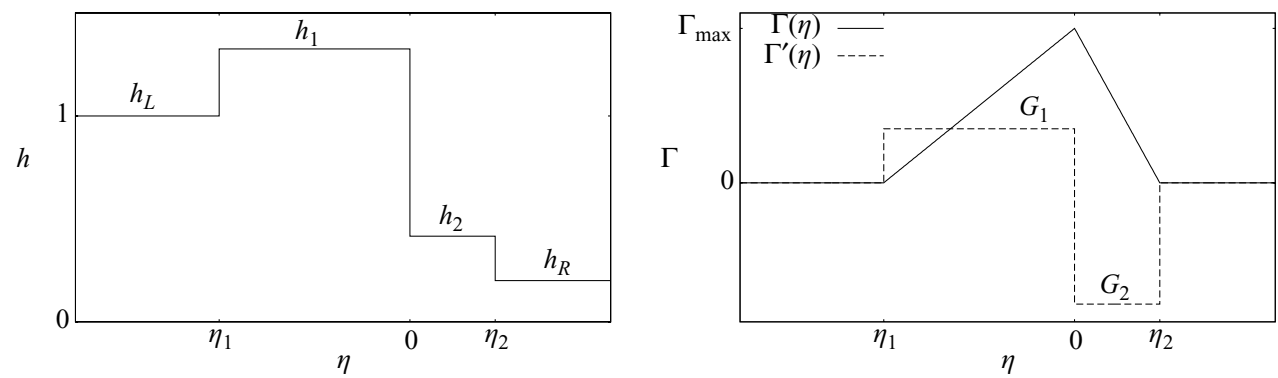

FIGURE 1. The piecewise constant weak traveling solution, (2.5): (left) $h(\eta)$ profile, (right) $\Gamma^{\prime}(\eta)$ profile (dashed), and the piecewise linear profile for $\Gamma(\eta),(2.6)$ (solid).

Provided that $h_{R} / h_{L}<q_{\text {crit }}$, a traveling wave in which $h$ and $\Gamma^{\prime}$ are piecewise constant functions can be constructed explicitly [14] (see Figure 1):

$$
h(\eta)=\left\{\begin{array}{ll}
h_{L} & \eta<\eta_{1} \\
h_{1} & \eta_{1}<\eta<0 \\
h_{2} & 0<\eta<\eta_{2} \\
h_{R} & \eta_{2}<\eta
\end{array} \quad \Gamma^{\prime}(\eta)= \begin{cases}0 & \eta<\eta_{1} \\
G_{1} & \eta_{1}<\eta<0 \\
G_{2} & 0<\eta<\eta_{2} \\
0 & \eta_{2}<\eta .\end{cases}\right.
$$

The surfactant concentration $\Gamma(\eta)$ then includes a constant of integration $\Gamma_{\max } \geqslant 0$ :

$$
\Gamma(\eta)= \begin{cases}0 & \eta \leqslant \eta_{1} \\ \Gamma_{\max }+G_{1} \eta & \eta_{1} \leqslant \eta \leqslant 0 \\ \Gamma_{\max }+G_{2} \eta & 0 \leqslant \eta \leqslant \eta_{2} \\ 0 & \eta_{2} \leqslant \eta\end{cases}
$$

in which $\eta_{1}, \eta_{2}$ are related to $\Gamma_{\max }$ so as to make $\Gamma(\eta)$ continuous,

$$
\eta_{1}=-\frac{\Gamma_{\max }}{G_{1}}<0, \quad \eta_{2}=-\frac{\Gamma_{\max }}{G_{2}}>0 .
$$

The total mass of surfactant for this solution is given by

$$
m=\int_{-\infty}^{\infty} \Gamma d \eta=\frac{1}{2} \Gamma_{\max }\left(\eta_{2}-\eta_{1}\right)=\frac{1}{2} \Gamma_{\max }^{2}\left(G_{1}^{-1}-G_{2}^{-1}\right) \geqslant 0
$$

Thus, for given upstream and downstream heights, either the mass or $\Gamma_{\max }$ may be used to parametrize the family of traveling waves. We refer to these traveling waves, in which $h$ is piecewise constant and $\Gamma$ is piecewise linear and continuous, as weak traveling wave solutions of system (2.1).

For a fixed upstream height $h_{L}$, the traveling waves exist for a range of downstream heights $h_{R}$. At the two extremes of this range, we get limiting profiles. In the limit as the downstream height $h_{R} \rightarrow 0^{+}$, the height $h(\eta)$ approaches a piecewise constant function with upstream height $h_{L}$, one intermediate height $h_{1}=\sqrt{2} h_{L}$ (since $h_{2} \rightarrow 0^{+}$), and $h_{R}=0$. The surfactant concentration $\Gamma(\eta)$ approaches a sawtooth function with $G_{1}=\sqrt{2} h_{L} / 3$ 


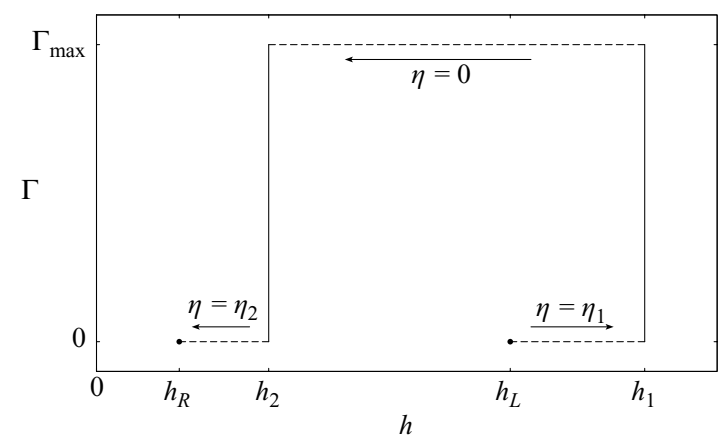

FIGURE 2. Representation of the weak solution in the $(h, \Gamma)$ phase plane. Horizontal (dashed) segments correspond to discontinuous jumps in $h$ and $\Gamma^{\prime}$ (corners of $\Gamma$ ) at the indicated spatial positions.

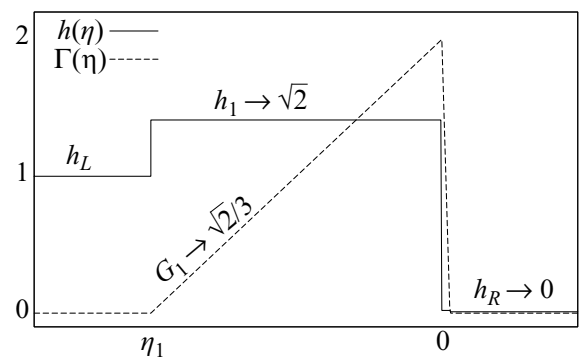

FIGURE 3. Approaching a degenerate profile for the limit $h_{R} \rightarrow 0$ : the $h_{2}$ plateau in the $h(\eta)$ profile (solid) vanishes with $\eta_{2}, h_{2} \rightarrow 0$ while the $\Gamma(\eta)$ profile (dashed) approaches a sawtooth shock wave, with $G_{2} \rightarrow-\infty$.

and $G_{2} \rightarrow-\infty$; the speed approaches $s=h_{L}^{2} / 3$. This limiting solution is shown in Figure 3 . A different solution emerges as $h_{R} / h_{L}$ approaches the critical ratio $q_{\text {crit }}$. In this case, the surfactant concentration vanishes uniformly with $G_{1} \rightarrow 0^{+}$and $G_{2} \rightarrow 0^{-}$, and the height of the film exhibits a single hydrodynamic jump from $h_{L}$ to $h_{R}=q_{\text {crit }} h_{L}$ with speed $s=\frac{1}{2} h_{L}^{2}$, since $h_{1}, h_{2} \rightarrow h_{L}$. This is a shock wave solution of the single equation (1.1) for the film height in the absence of surfactant.

\subsection{Stability analysis}

There are several significant speeds associated with system (2.1) and the solution (2.5). The speed $s$ of the traveling wave is the speed of the discontinuities in $h, \Gamma_{x}$. While $(2.1 a b)$ is not a standard hyperbolic system, we can still obtain some insight into stability by linearizing about the piecewise weak solution. We consider infinitesimal perturbations to $h(\eta)$ and $\Gamma(\eta)$ in the reference frame moving with the traveling wave,

$$
h \sim h(\eta)+\tilde{h}(\eta, t), \quad \Gamma \sim \Gamma(\eta)+\tilde{\Gamma}(\eta, t) .
$$




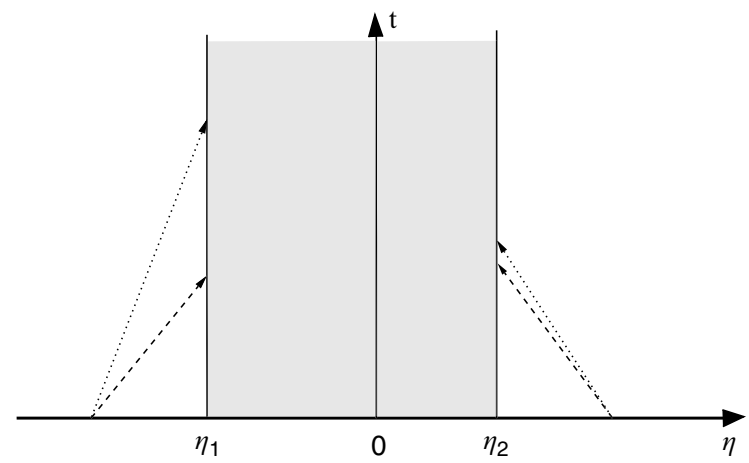

FIGURE 4. Wave speeds and parabolic regions in the reference frame moving with the weak traveling wave: $\eta=x-s t$. Dashed lines: characteristic speed $c$; dotted lines: speed $v$ of perturbations.

The linearized system for $\tilde{h}(\eta, t), \tilde{\Gamma}(\eta, t)$ is

$$
\begin{aligned}
& \tilde{h}_{t}+c \tilde{h}_{\eta}=\frac{1}{2} h^{2} \tilde{\Gamma}_{\eta \eta}, \\
& \tilde{\Gamma}_{t}+v \tilde{\Gamma}_{\eta}+w \eta \tilde{h}_{\eta}=h G \eta \tilde{\Gamma}_{\eta \eta}-w \tilde{h},
\end{aligned}
$$

with

$$
c=h^{2}-h G-s, \quad v=\frac{1}{2} h^{2}-2 h G-s, \quad w=h G-G^{2},
$$

where the $h$ and $G$ appearing in the coefficients and speeds are constant in each of the four intervals $\{L, 1,2, R\}$, comprising the weak solution (2.5), where we set $G_{L}=G_{R}=0$. In general (2.9) is a parabolic system, but in the intervals $L, R$ we have $G=0$ and it reduces to

$$
\begin{aligned}
& \tilde{h}_{t}+c \tilde{h}_{\eta}=\frac{1}{2} h^{2} \tilde{\Gamma}_{\eta \eta}, \\
& \tilde{\Gamma}_{t}+v \tilde{\Gamma}_{\eta}=0 .
\end{aligned}
$$

This linear system has the general solution

$$
\tilde{h}(\eta, t)=f\left(\eta-\left[h^{2}-s\right] t\right)+g^{\prime}\left(\eta-\left[\frac{1}{2} h^{2}-s\right] t\right), \quad \tilde{\Gamma}(\eta, t)=g\left(\eta-\left[\frac{1}{2} h^{2}-s\right] t\right),
$$

in which $f(\zeta), g(\xi)$ are arbitrary functions describing two independent traveling waves. For $h_{R} / h_{L}<q_{\text {crit }}$, both families of waves propagate inward toward the shocks at $\eta=\eta_{1}$ and $\eta=\eta_{2}$ from intervals $L$ and $R$ respectively, see Figure 4. Hence these two shocks form a structure that is overcompressive: small disturbances in both $h$ and $\Gamma$ approach the wave from both ahead and behind the wave. The term overcompressive derives from systems of hyperbolic conservation laws [26]; although our equations are hyperbolic/degenerateparabolic, the term is appropriate here too. Equation (1.1) has classical compressive shock solutions with a single set of characteristics entering from either side of the shock. Undercompressive shocks also occur in thin film flow [4] and have all families of characteristics passing through the shock. 

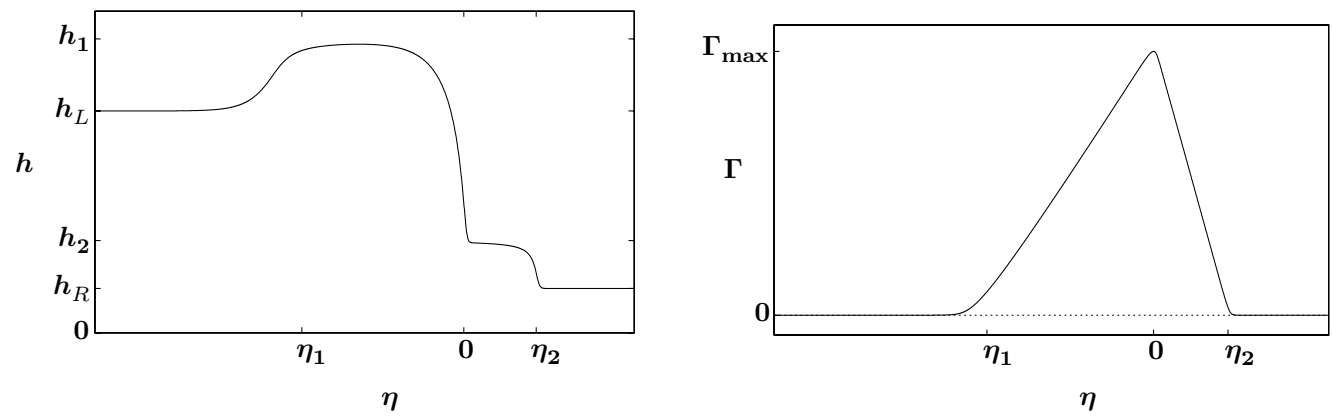

Figure 5. A typical traveling wave solution, $h(\eta)$ (left) and $\Gamma(\eta)$ (right), with second-order regularization, $\beta, \delta>0$.

The overcompressive nature of the weak solution is also reflective of its nonuniqueness, subject to fixed boundary conditions $h_{L}, h_{R}$. Whereas undercompressive waves appear only for discrete values of a parameter, such as $h_{L}$ [4], overcompressive waves occur in continuous one-parameter families for a range of boundary conditions (here for all $\Gamma_{\max }>0$ at each fixed $\left.h_{L}, h_{R}\right)$.

Unlike the other two shocks, the stability of the shock at $\eta=0$ must be studied through the coupled parabolic system (2.9) on intervals either side of the shock. ${ }^{1}$ Hence we might expect it to have different properties than the outer pair. Indeed we will see that adding regularizing terms to the system does influence these shocks differently, but preserves the overcompressive property of having a one-parameter family of solutions.

\section{Traveling waves with second-order regularization}

In this section we consider the form of traveling wave solutions in the presence of the second-order regularizing terms from (1.9ab). That is, we take $\kappa=0$ and $\beta, \delta>0$ to yield a second order system of ODEs,

$$
\begin{gathered}
-s h+\frac{1}{3} h^{3}-\frac{1}{2} h^{2} \frac{d \Gamma}{d \eta}=\frac{1}{3} \beta h^{3} \frac{d h}{d \eta}-k_{h}, \\
-s \Gamma+\frac{1}{2} h^{2} \Gamma-h \Gamma \frac{d \Gamma}{d \eta}=\frac{1}{2} \beta h^{2} \Gamma \frac{d h}{d \eta}+\delta \frac{d \Gamma}{d \eta} .
\end{gathered}
$$

A typical solution is shown in Figure 5. Relative to the weak solution from Section 2, the solution here has been smoothed at its jumps at $\eta=\eta_{1}, 0, \eta_{2}$. We describe the structure of $(3.1 \mathrm{ab})$ via phase plane analysis and then consider the smoothing effects introduced by the $\beta, \delta$ parameters.

\subsection{Phase plane analysis for general $\beta, \delta>0$}

Equations (3.1ab) can be written as a first-order autonomous system in the plane:

$$
\begin{gathered}
\beta \frac{d h}{d \eta}=\frac{P_{1}(h) \Gamma h+4 \delta P_{0}(h)}{h^{3}(\Gamma h+4 \delta)} \\
\frac{d \Gamma}{d \eta}=\frac{2 s \Gamma\left(h-h_{*}\right)}{h(\Gamma h+4 \delta)},
\end{gathered}
$$

1 We do not analyze this problem here. 

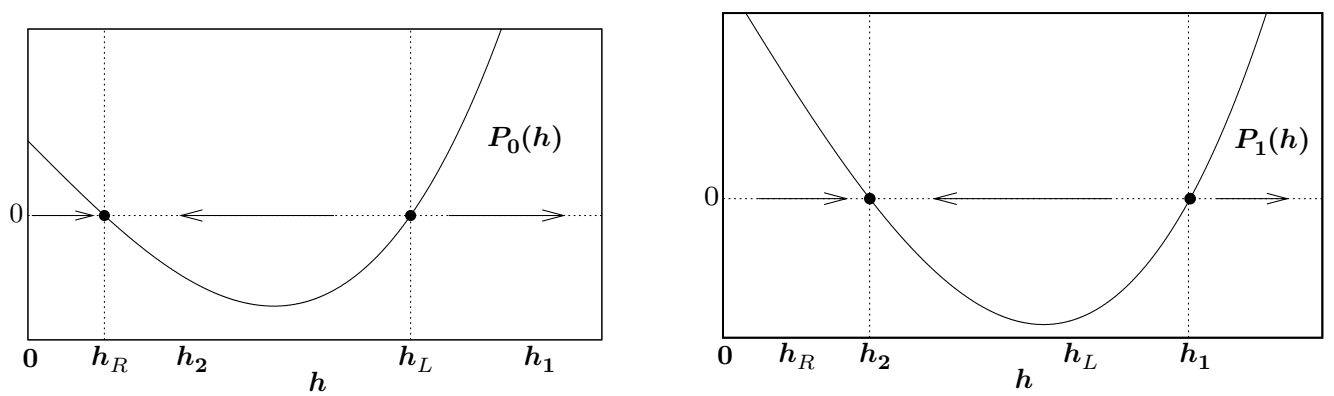

Figure 6. (Left) The polynomial $P_{0}(h)$ and the direction field for monotone increasing/decreasing solutions on the $h$-axis, with no surfactant, equation (3.10). (Right) The $P_{1}(h)$ polynomial and the direction field associated with equation (3.11).

where the constant $h_{*}$ in $(3.2 b)$ is $h_{*}=3 k_{h} / s$, i.e.,

$$
h_{*}=\frac{3 h_{L} h_{R}\left(h_{L}+h_{R}\right)}{h_{L}^{2}+h_{L} h_{R}+h_{R}^{2}}
$$

and the cubic polynomials $P_{0}(h), P_{1}(h)$ in $(3.2 a)$ are

$$
P_{0}(h)=h^{3}-3 s h+3 k_{h}, \quad P_{1}(h)=h^{3}-6 s h+12 k_{h} .
$$

Observe that $P_{0}(h)$ factors as

$$
P_{0}(h)=\left(h-h_{L}\right)\left(h-h_{R}\right)\left(h+h_{L}+h_{R}\right) .
$$

Noting that equation (2.3) for the heights $h_{1}$ and $h_{2}$ corresponds to $P_{1}(h)=0$ from (3.4), we can similarly factor $P_{1}$ when $h_{R} / h_{L}<(\sqrt{3}-1) / 2$,

$$
P_{1}(h)=\left(h-h_{1}\right)\left(h-h_{2}\right)\left(h+h_{1}+h_{2}\right) .
$$

The ordering of the zeros of $P_{0}(h)$ and $P_{1}(h)$ is given in the following lemma (see Figure 6).

Lemma 3.1 For $0<h_{R} / h_{L}<(\sqrt{3}-1) / 2$, the positive zeroes $h_{L}, h_{R}$ of $P_{0}(h)$ and $h_{1}, h_{2}$ of $P_{1}(h)$ respectively are ordered as

$$
h_{R}<h_{2}<h_{*}<h_{L}<h_{1} \text {, }
$$

where $h_{*}$ is given by (3.3).

Proof We first show that $h_{R}<h_{*}<h_{L}$. Consider the difference $h_{L}-h_{*}$; it can be written in the form

$$
h_{L}-h_{*}=\frac{h_{L}^{3}}{h_{L}^{2}+h_{L} h_{R}+h_{R}^{2}}\left(1-2 q-2 q^{2}\right) \text {, }
$$



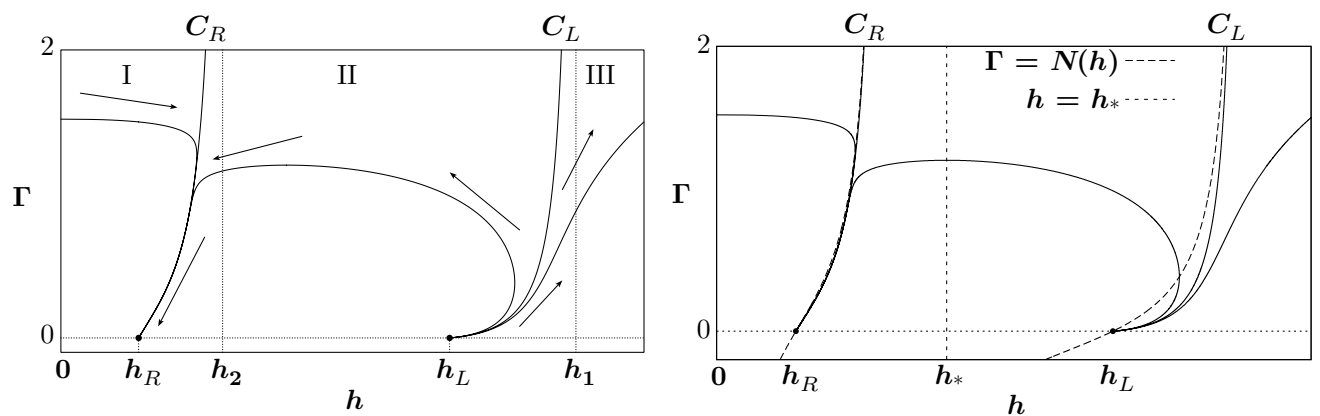

FiguRE 7. The $(h, \Gamma)$ phase portrait of system $(3.2 a b)$ for $\beta, \delta>0$ : (Left) Equilibria $\left(h_{L}, 0\right),\left(h_{R}, 0\right)$, some representative solution curves with arrows indicating the direction of flow with $\eta$. The singular trajectories $C_{L}, C_{R}$ partition the space into three regions with bounded heteroclinic orbits existing in region II. (Right) Trajectories in the phase plane along with the nullclines (dashed curves).

where $q=h_{R} / h_{L}$. The prefactor is positive and the quadratic is positive for $0<q<$ $(\sqrt{3}-1) / 2$. Hence $h_{L}>h_{*}$. Similarly,

$$
h_{*}-h_{R}=\frac{h_{R} h_{L}^{2}}{h_{L}^{2}+h_{L} h_{R}+h_{R}^{2}}\left(2+2 q-q^{2}\right)>0
$$

over the wider range $0<q<1+\sqrt{3}$; hence $h_{*}>h_{R}$. It now follows from (3.5) that $P_{0}\left(h_{*}\right)<0$. Moreover, since $k_{h}=\frac{1}{3} s h_{*}, P_{1}(h)-P_{0}(h)=3 s\left(h_{*}-h\right)$, from (3.4). Therefore, $P_{1}\left(h_{*}\right)=P_{0}\left(h_{*}\right)<0$. Hence, from (3.6) $h_{2}<h_{*}<h_{1}$. Since $P_{0}\left(h_{R}\right)=0$, $P_{1}\left(h_{R}\right)=P_{1}\left(h_{R}\right)-P_{0}\left(h_{R}\right)=3 s\left(h_{*}-h_{R}\right)>0$, so $h_{2}>h_{R}$. Similarly, at $h_{L}, P_{0}\left(h_{L}\right)=0$, so $P_{1}\left(h_{L}\right)=P_{1}\left(h_{L}\right)-P_{0}\left(h_{L}\right)=3 s\left(h_{*}-h_{L}\right)<0$, so that $h_{1}>h_{L}$.

Physically relevant solutions are contained in the first quadrant, $h \geqslant 0, \Gamma \geqslant 0$; some representative trajectories are shown in Figure 7 . There are two equilibria: $\left(h_{L}, 0\right)$ and $\left(h_{R}, 0\right)$. The equilibrium $\left(h_{L}, 0\right)$ is an unstable node with eigenvalues

$$
\lambda_{1}=\frac{\left(h_{L}-h_{R}\right)\left(2 h_{L}+h_{R}\right)}{\beta h_{L}^{3}}>0, \quad \lambda_{2}=\frac{s\left(h_{L}-h_{*}\right)}{2 \delta h_{L}}>0 .
$$

The equilibrium $\left(h_{R}, 0\right)$ is a stable node with eigenvalues

$$
\lambda_{1}=-\frac{\left(h_{L}-h_{R}\right)\left(2 h_{R}+h_{L}\right)}{\beta h_{R}^{3}}<0, \quad \lambda_{2}=\frac{s\left(h_{R}-h_{*}\right)}{2 \delta h_{R}}<0 .
$$

The global structure of the phase plane can be understood by considering limiting behaviors for $\Gamma$. If there is no surfactant, system (3.1ab) reduces to a single equation for $h(\eta)$, on the $\Gamma \equiv 0$ invariant line

$$
\frac{d h}{d \eta}=\frac{P_{0}(h)}{\beta h^{3}} \quad \text { for } \Gamma=0,
$$


see Figure 6 (left). In the other extreme, for $\Gamma \rightarrow \infty$, from $(3.2 a)$, if $h$ is bounded it must satisfy

$$
\frac{d h}{d \eta}=\frac{P_{1}(h)}{\beta h^{3}} \quad \text { for } \Gamma \rightarrow \infty .
$$

Consequently, we note the existence of two singular solutions, $C_{L}$ which connects $\left(h_{L}, 0\right)$ to $\left(h_{1}, \infty\right)$, and $C_{R}$ which connects $\left(h_{2}, \infty\right)$ to $\left(h_{R}, 0\right)$, see Figure 7 . These trajectories are separatrices that partition the phase plane into three regions, labeled I, II, III in Figure 7. All solutions in regions I and II approach $\left(h_{R}, 0\right)$ as $\eta \rightarrow \infty$. All solutions in regions II and III approach $\left(h_{L}, 0\right)$ as $\eta \rightarrow-\infty$. From region II, any nonnegative bounded solutions must be heteroclinic orbits connecting the equilibria.

From $(3.2 b)$, the $\Gamma$-nullcline, where $d \Gamma / d \eta=0$, is given by the line $h=h_{*}$. Hence, the value of $\Gamma$ where $h=h_{*}$ is the local (and in region II, the global) maximum of $\Gamma(\eta)$. This gives us a very convenient parametrization of all the solutions in region II.

Theorem 3.2 Every heteroclinic orbit from $\left(h_{L}, 0\right)$ to $\left(h_{R}, 0\right)$ corresponds to a solution of (3.2ab) with

$$
h(0)=h_{*}, \quad \Gamma(0)=\Gamma_{\max },
$$

for some $\Gamma_{\max } \geqslant 0$. Conversely, for every $\Gamma_{\max } \geqslant 0$, there is a heteroclinic orbit from $\left(h_{L}, 0\right)$ to $\left(h_{R}, 0\right)$.

Proof Every heteroclinic orbit $(h(\eta), \Gamma(\eta))$ from $\left(h_{L}, 0\right)$ to $\left(h_{R}, 0\right)$ crosses $h=h_{*}$ at some value $\Gamma_{\max }$ of $\Gamma$. After a suitable translation of the independent variable $\eta$, this trajectory satisfies (3.12).

Conversely, as described above, all bounded solutions are heteroclinic orbits in region II of the phase plane, connecting $\left(h_{L}, 0\right)$ as $\eta \rightarrow-\infty$ and $\left(h_{R}, 0\right)$ as $\eta \rightarrow \infty$. Since (3.12) is an ordinary point for the autonomous system (3.2ab), there exists a unique trajectory that passes through it. Consequently (3.12) defines a heteroclinic solution in region II for all positive $\Gamma_{\max }$.

From (3.2a), the $h$-nullcline, where $d h / d \eta=0$, is the graph of the rational function,

$$
\Gamma=N(h) \equiv-\frac{4 \delta P_{0}(h)}{h P_{1}(h)}
$$

$N(h)$ has zeroes at $h=h_{L}, h=h_{R}$ and vertical asymptotes at $h=h_{1}, h=h_{2}$, see Figure 7(right). As shown in Figure 7, the separatrices $C_{L}, C_{R}$ lie to the right of the components of the $h$ nullcline through $\left(h_{L}, 0\right),\left(h_{R}, 0\right)$, respectively. Trajectories intersecting the nullcline at positive $\Gamma$ have nonmonotone $h(\eta)$ solution profiles. Using (3.7), it can be shown that the slope of the nullcline at the equilibrium points, $h_{R}$ and $h_{L}$, is positive, $N^{\prime}\left(h_{L, R}\right)>0$. We can determine if heteroclinic solutions intersect the nullcline by considering their linearized behavior at the equilibria. The eigenvectors corresponding to 
eigenvalues $\lambda_{1}, \lambda_{2}(3.8)$ are

$$
\mathbf{v}_{1}=\left(\begin{array}{l}
1 \\
0
\end{array}\right), \quad \mathbf{v}_{2}=\left(\begin{array}{c}
-h_{L} P_{1}\left(h_{L}\right) \\
4 \delta\left(h_{L}-h_{R}\right)\left(2 h_{L}+h_{R}\right)-2 \beta s h_{L}^{2}\left(h_{L}-h_{*}\right)
\end{array}\right) .
$$

Hence the linearized solutions for $\eta \rightarrow-\infty$ take the form

$$
\begin{aligned}
h(\eta) & \sim h_{L}+c_{1} e^{\lambda_{1} \eta}-c_{2} h_{L} P_{1}\left(h_{L}\right) e^{\lambda_{2} \eta} \\
\Gamma(\eta) & \sim c_{2}\left[4 \delta\left(h_{L}-h_{R}\right)\left(2 h_{L}+h_{R}\right)-2 \beta s h_{L}^{2}\left(h_{L}-h_{*}\right)\right] e^{\lambda_{2} \eta} .
\end{aligned}
$$

The eigenvector $\mathbf{v}_{1}$ is parallel to the $\Gamma \equiv 0$ invariant line. The slope associated with the eigenvector $\mathbf{v}_{2}$ is positive if

$$
\frac{\beta}{\delta} \leqslant \rho_{L} \equiv \frac{2\left(h_{L}-h_{R}\right)\left(2 h_{L}+h_{R}\right)}{s h_{L}^{2}\left(h_{L}-h_{*}\right)} .
$$

Note that this condition is equivalent to

$$
\lambda_{1} \geqslant \lambda_{2}
$$

for the eigenvalues in (3.8). That is, as $\eta \rightarrow-\infty$ the $e^{\lambda_{2} \eta}$ contributions in (3.15) decay more slowly and ultimately dominate the asymptotic behavior as the equilibrium is approached. Consequently, solution trajectories with $\Gamma>0$ (i.e. $\left.c_{2}>0\right)$ will approach $\left(h_{L}, 0\right)$ along the $\mathbf{v}_{2}$ vector in the phase plane. Noting that $P_{1}\left(h_{L}\right)<0$, given any value of $c_{1}$, for sufficiently large $|\eta|$, as $\eta \rightarrow-\infty, h(\eta)$ will approach $h_{L}$ from above. Since $h(0)=h_{*}<h_{L}$, we conclude that such solutions are nonmonotone and must cross the $h$-nullcline (see Figure 8 (left)).

If condition (3.16) is violated, the slope corresponding to eigenvector $\mathbf{v}_{2}$ is negative, and since $e^{\lambda_{1} \eta}>e^{\lambda_{2} \eta}$ for $\eta<0$, trajectories enter $\left(h_{L}, 0\right)$ tangent to $\mathbf{v}_{1}$, i.e., tangent to the $h$-axis, unless $c_{1}=0$ in (3.15) (in which case, the trajectory is tangent to $\mathbf{v}_{2}$ ). Correspondingly, there is a finite range of values of $\Gamma_{\max }, 0 \leqslant \Gamma_{\max } \leqslant \Gamma_{\max }^{\text {crit }}(\beta / \delta)$, for which the trajectory of Theorem 3.2 is monotonic decreasing. For $\Gamma_{\max }=\Gamma_{\max }^{\operatorname{crit}}(\beta / \delta)$, the trajectory has $c_{1}=0$ in (3.15) and is still monotonic. For $\Gamma_{\max }>\Gamma_{\max }^{\mathrm{crit}}(\beta / \delta)$, the trajectory crosses the $h$-nullcline and is nonmonotonic as before. These features are shown in Figure 8. The $C_{L}$ separatrix has similar behavior. At the equilibrium $\left(h_{L}, 0\right), C_{L}$ is tangent to $\mathbf{v}_{1}$ if $\beta / \delta<\rho_{L}$, but is tangent to the $h$ axis (with $h>h_{L}$ ) if $\beta / \delta>\rho_{L}$.

Similar to (3.16), the asymptotic behavior as $\eta \rightarrow \infty$ involves a critical ratio relating the regularizing parameters to the eigenvalues (3.9), i.e., $\lambda_{1} \geqslant \lambda_{2}$ :

$$
\frac{\beta}{\delta} \leqslant \rho_{R} \equiv \frac{2\left(h_{R}-h_{L}\right)\left(2 h_{R}+h_{L}\right)}{s h_{R}^{2}\left(h_{R}-h_{*}\right)} .
$$

Similar to the above arguments, the singular trajectory $C_{R}$ is bounded between the nullcline and the asymptote $h=h_{2}$. In this case, although the separatrix $C_{R}$ arrives at $\left(h_{R}, 0\right)$ along either the $\mathbf{v}_{2}$ or $\mathbf{v}_{1}$ eigenvector depending on whether (3.17) is satisfied, this does not lead to a change in the monotonicity of $h(\eta)$ as $h_{R}$ is approached; all solutions are monotone decreasing as they enter the equilibrium $\left(h_{R}, 0\right)$.

In the following subsections, we use asymptotic analysis to study the influence of weak regularization on the ODE system (3.1ab), i.e., the limits $\delta \rightarrow 0$ and $\beta \rightarrow 0$. The $\delta, \beta$ 

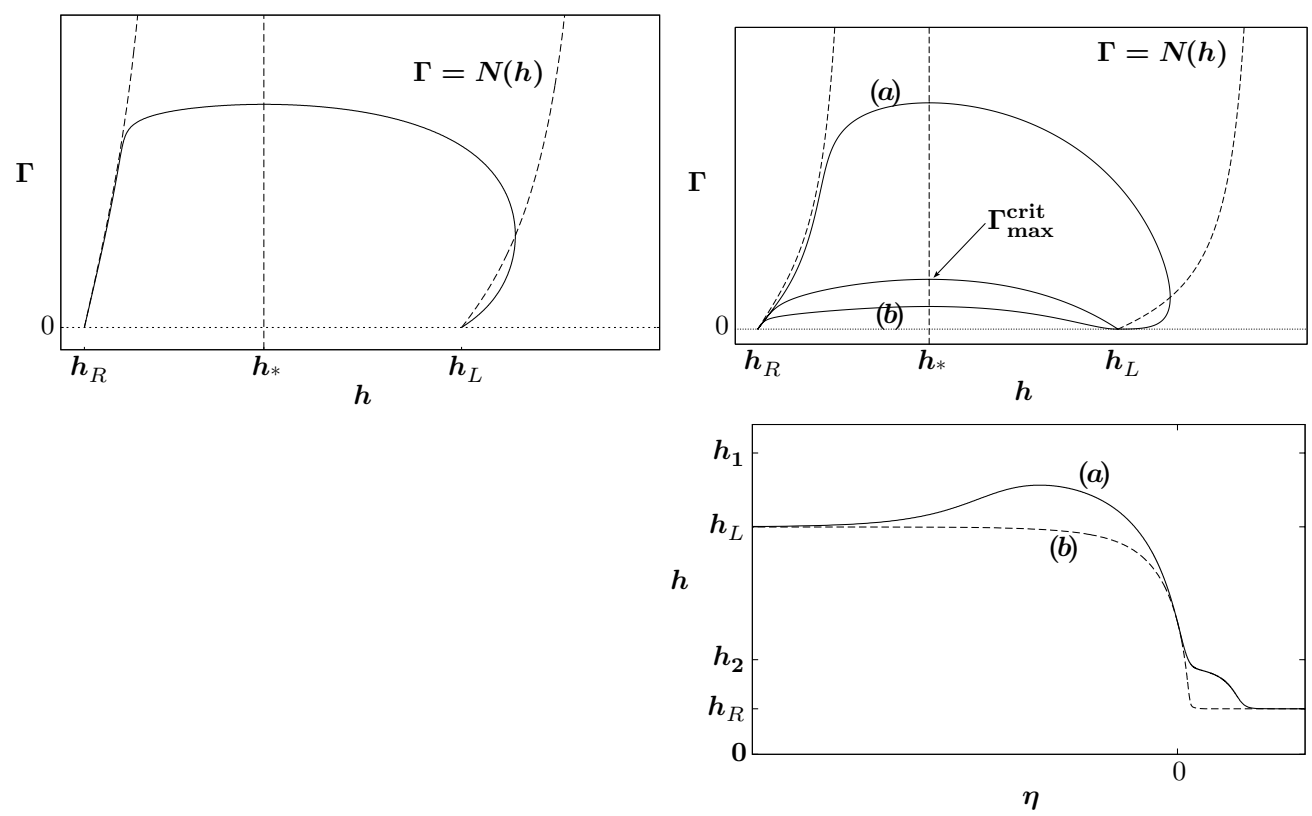

FigURE 8. Influence of parameters $\beta, \delta$ on heteroclinic solutions: (Left) for $\beta / \delta<\rho_{L}$ all bounded solutions cross the right nullcline and yield nonmonotone $h(\eta)$ profiles (see Figure 5 for example), (Right) for $\beta / \delta>\rho_{L}$ there is a critical value $\Gamma_{\max }^{\text {crit }}(\beta / \delta)$ that separates monotone-in- $h$ and nonmonotone solutions, see lower-right for two such typical $h(\eta)$ profiles.

terms introduce singular perturbations and smoothing that fundamentally change aspects of the structure of the solutions. For example, consider the behavior near the equilibria $\left(h_{L}, 0\right),\left(h_{R}, 0\right)$; the $\lambda_{2}$ eigenvalues (see (3.8), (3.9)) diverge such as $O(1 / \delta) \rightarrow \infty$, as $\delta \rightarrow 0$, and the $\lambda_{1}$ eigenvalues diverge such as $O(1 / \beta) \rightarrow \infty$ as $\beta \rightarrow 0$. The behavior with these singular eigenvalues as $|\eta| \rightarrow \infty$, suggests that these contributions to the solutions reach zero at finite $\eta$. From (3.15), $\Gamma(\eta)$ should approach a function with compact support as $\delta \rightarrow 0$, since it involves only $\lambda_{2}$, while $h(\eta)$ has exponential decay for $|\eta| \rightarrow \infty$ unless $\delta=\beta=0$. In fact, as remarked in Section 5, solutions with $\delta=0$ have this property. In the following subsections, we use matched asymptotics to describe the structure of the solution for three interesting limits. In presenting asymptotic expansions for solutions in these various parameter regimes, we use the symbol $z$ to denote a scaled independent spatial variable in a variety of contexts in order to avoid a proliferation of symbols. The meaning of $z$ is consistent within each subsection.

\subsection{Solution for the $\Gamma_{\max } \rightarrow \infty$ limit}

We briefly consider the structure of the solutions for fixed $\beta, \delta>0$ as $\Gamma_{\max } \rightarrow \infty$. This limit was also studied by the authors for a problem with a quasi-statically growing traveling wave, driven by a steady rate of influx of surfactant [31].

Define $\sigma=1 / \Gamma_{\max }$ as the small parameter, for this case $\sigma \rightarrow 0$. Recalling (2.6) and (2.7), the magnitude (and region of support if $\delta=0$ ) of the surfactant profile can be expected to scale with $\sigma$, see Figure 9. Hence, in this section we define $z=\sigma \eta$ and we rescale the 

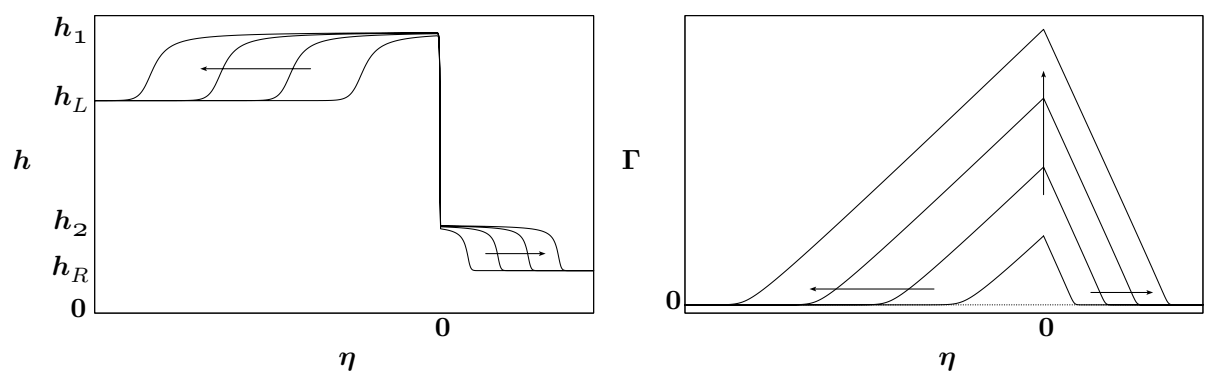

FIGURE 9. Numerical solutions for $h(\eta), \Gamma(\eta)$ for several values of $\Gamma_{\max }$ illustrating the trends for for the limit $\sigma \rightarrow 0\left(\Gamma_{\max } \rightarrow \infty\right)$. Here $\delta=0.1, \beta=0.1, h_{L}=1$ and $h_{R}=0.2$ with $\Gamma_{\max }=5,10,15,20$.

solution as

$$
h=\check{h}(z), \quad \Gamma=\frac{1}{\sigma} \check{\Gamma}(z), \quad \eta=\frac{z}{\sigma},
$$

where $\check{\Gamma}(0)=1$ corresponds to the condition $\Gamma(0)=\Gamma_{\max }$ of Theorem 3.2. The governing equations then become

$$
\frac{d \check{h}}{d z}=\frac{P_{1}(\check{h}) \check{\Gamma} \check{h}+4 \sigma \delta P_{0}(\check{h})}{\sigma \beta \check{h}^{3}(\check{\Gamma} \check{h}+4 \sigma \delta)}, \quad \frac{d \check{\Gamma}}{d z}=\frac{2 s \check{\Gamma}\left(\check{h}-h_{*}\right)}{\check{h}(\check{\Gamma} \check{h}+4 \sigma \delta)} .
$$

Writing the solution as a perturbation expansion,

$$
\check{h}=\check{h}^{0}(z)+\sigma \check{h}^{1}(z)+O\left(\sigma^{2}\right), \quad \check{\Gamma}=\check{\Gamma}^{0}(z)+\sigma \check{\Gamma}^{1}(z)+O\left(\sigma^{2}\right),
$$

and substituting this ansatz into (3.2a) yields that at leading order $\sigma^{-1} P_{1}\left(\breve{h}^{0}\right)=0$. Therefore, to leading order, the film height is one of the roots, $\check{h}^{0}=h_{1}$ or $\check{h}^{0}=h_{2}$ of $P_{1}$. At leading order for $\check{\Gamma}$ we find corresponding constant slopes given by

$$
\frac{d \check{\Gamma}^{0}}{d z}=\frac{2 s\left(\check{h}^{0}-h_{*}\right)}{\left(\check{h}^{0}\right)^{2}} .
$$

Using algebraic relations from [14], we recognize these slopes as being equivalent to $G_{1}, G_{2}$ respectively (see (2.4)). Consequently the piecewise-defined leading order solution is

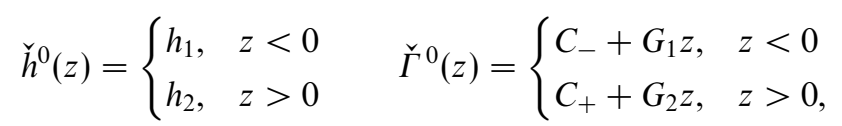

where $C_{ \pm}$are constants. We show below that these constants in the outer solution are determined, by matching to the inner solution, to be $C_{-}=C_{+}=1$, so (3.22) recovers the weak traveling wave solution (2.5), (2.6) on the interval where $\check{\Gamma}^{0}>0$, see Figure 10(right).

At next order in $\sigma$, we obtain an algebraic equation

$$
\check{h}^{1}(z)=-\frac{4 \delta P_{0}\left(\check{h}^{0}\right)}{\check{h}^{0} P_{1}^{\prime}\left(\check{h}^{0}\right) \check{\Gamma}^{0}(z)},
$$



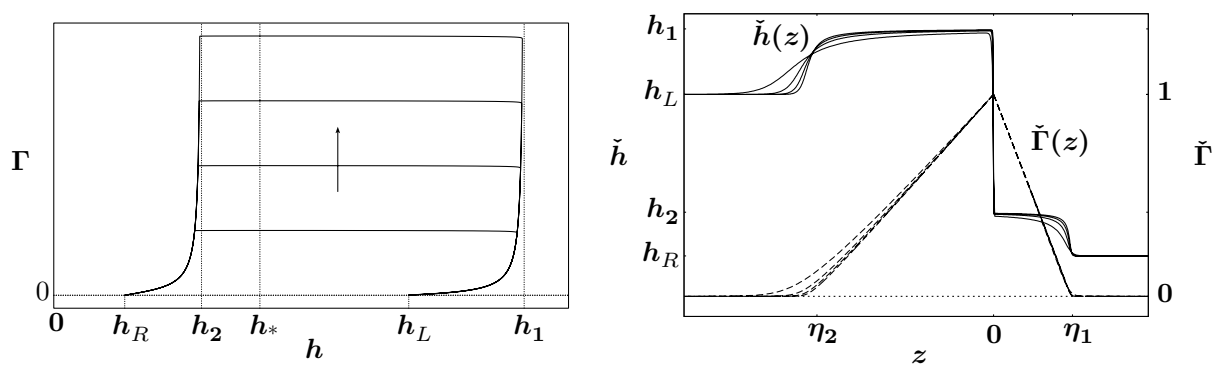

FIGURE 10. (Left) Traveling waves in the $h, \Gamma$ phase plane for $\sigma \rightarrow 0$, (Right) rescaled outer solutions (3.18) for the profiles in Figure 9.

determining $\check{h}^{1}(z)$, with $\check{h}^{1}(|z| \rightarrow \infty)=O\left(|z|^{-1}\right) \rightarrow 0$. Likewise, higher order corrections to $\check{\Gamma}(z)$ decay algebraically as $|z| \rightarrow \infty$.

For $\beta, \delta>0$, we expect the solutions ( $\breve{h}(z)$ in particular) to be smooth and continuous, hence (3.22) cannot be valid at $z=0$. To describe the solution structure near $\eta=0$, we consider perturbation expansions in the original variables (which are inner variables relative to (3.18)),

$$
h(\eta)=h^{0}(\eta)+\sigma h^{1}(\eta)+O\left(\sigma^{2}\right) \quad \Gamma(\eta)=\frac{1}{\sigma}+\Gamma^{1}(\eta)+\sigma \Gamma^{2}(\eta)+O\left(\sigma^{2}\right) .
$$

with conditions $h^{0}(0)=h_{*}$ and $h^{n}(0)=\Gamma^{n}(0)=0$ for $n \geqslant 1$. To leading order as $\sigma \rightarrow 0$, (3.2ab) reduce to

$$
\frac{d h^{0}}{d \eta}=\frac{P_{1}\left(h^{0}\right)}{\beta\left(h^{0}\right)^{3}}, \quad \frac{d \Gamma^{1}}{d \eta}=\frac{2 s\left(h^{0}-h_{*}\right)}{\left(h^{0}\right)^{2}} .
$$

We note that the structure of this interior boundary layer depends to leading order on $\beta$; this dependence will be explored further in Section 3.3. The parameter $\delta$ comes in at higher orders and hence has a weak influence on the structure of the solution near $\eta=0$. The equation for $h^{0}(\eta)$ is decoupled from $\Gamma^{1}$ and describes a unique monotone-decreasing solution from $h_{1}$ to $h_{2}$. Continuing to $O(\sigma)$, from asymptotic matching of $h^{1}(\eta \rightarrow \pm \infty)$ to $\check{h}^{1}\left(z \rightarrow 0^{ \pm}\right)$, we indeed obtain that $\check{\Gamma}^{0}\left(0^{ \pm}\right)=1$ for (3.22), establishing that $\check{\Gamma}$ is continuous and has max 1 .

Outside of the interval $\left(\eta_{1}, \eta_{2}\right), \Gamma(\eta)$ is no longer large and a different representation is more appropriate, see Section 3.4. For $\eta \leqslant \eta_{1}$ and $\eta \geqslant \eta_{2}, h(\eta)$ and $\Gamma(\eta)$ are $O(1)$; the structure of the solution describing the connections to $\Gamma \rightarrow 0$ and $h \rightarrow h_{L}, h \rightarrow h_{R}$ are given by the full equations, $(3.2 a b)$.

We see that in the limit $\Gamma_{\max } \rightarrow \infty$ the separations between 0 and $\eta_{1}, 0$ and $\eta_{2}$ grow linearly with $\Gamma_{\max }$ and hence the localized structures of the solution in these neighborhoods become more independent and clearly defined. In contrast, for $\Gamma_{\max }<\Gamma_{\max }^{\text {crit }}$ (defined in Section 3.1), these positions are so closely spaced that the regularization due to $\beta, \delta$ completely eliminates the $h_{1}, h_{2}$ plateaus (recall curve (b) in Figure 8 (lower right)). In other words, sufficiently large concentrations of surfactant (as measured by $\Gamma_{\max }$ ) are necessary to generate identifiable $h_{1}, h_{2}$ height plateaus. 

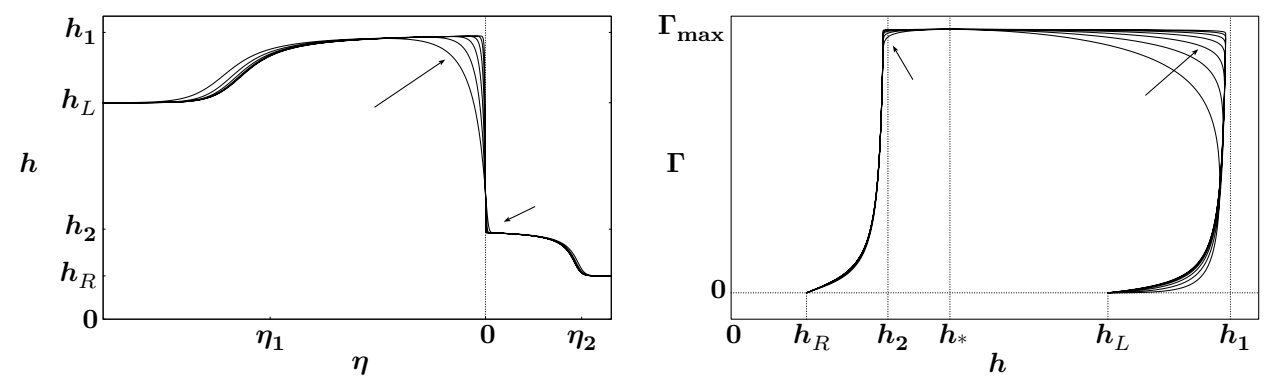

FIgURE 11. Trends for $\beta \rightarrow 0$. The dominant effect is on the structure of the jump in $h, \Gamma^{\prime}$ at $\eta=0$, shown in the $h(\eta)$ profile (left) and in the $h, \Gamma$ phase plane (right). Here $h_{L}=1, h_{R}=0.2, \delta=0.025$ and $\Gamma_{\max }=2$ with $\beta=2^{-n}$ for $n=0,1,2 \ldots 6$.

\subsection{Solution for the $\beta \rightarrow 0$ limit}

Figure 11 illustrates the trends seen in numerically computed solutions in the limit of $\beta \rightarrow 0$ with fixed positive $\delta$. The dominant effect is in decreasing the width of the shock layer in $h$ at $\eta=0$. We now present analysis to support this observation.

The limit $\beta \rightarrow 0$ is a singular limit of $(3.2 a b)$, since it reduces the ODE for $h(\eta)$ to an algebraic relation. To leading order, with $h \sim h^{0}(\eta), \Gamma \sim \Gamma^{0}(\eta)$, we obtain the differential-algebraic system

$$
\Gamma^{0}=N\left(h^{0}\right), \quad \frac{d \Gamma^{0}}{d \eta}=\frac{2 s \Gamma^{0}\left(h^{0}-h_{*}\right)}{h^{0}\left(\Gamma^{0} h^{0}+4 \delta\right)},
$$

where $N(h)$ is given by (3.13). Hence, the leading order outer solution is defined in terms of the $h$-nullcline (3.13). It is convenient to re-write this problem as a first order ODE for $h^{0}(\eta)$ by substituting $N\left(h^{0}\right)$ into the ODE for $\Gamma^{0}$,

$$
\frac{d h^{0}}{d \eta}=M\left(h^{0}\right), \quad M(h) \equiv \frac{2 s N(h)\left(h-h_{*}\right)}{h N^{\prime}(h)(h N(h)+4 \delta)} .
$$

This form makes it clear that (3.25) can produce monotone smooth solutions for $\left(h^{0}, \Gamma^{0}\right)$ defined on ranges of $h$ between the zeroes of $M(h)$. In particular, we obtain piecewise outer solutions defining a monotone increasing $h^{0}(\eta)$ for $h_{L} \leqslant h^{0}<h_{1}$ on $\eta<0$ and a monotone decreasing portion for $h_{R} \leqslant h^{0}<h_{2}$ on $\eta>0$, (see Figure 12). Since $N(h)$ has positive slope on these intervals, $\Gamma^{0}(\eta)$ has the same monotonicity as $h^{0}(\eta)$.

However, the solution of (3.25) cannot connect the two portions of the outer solution across $\eta=0$ since the $h$-nullcline gives a clearly unphysical negative surfactant concentration, $N(h)<0$, for $h_{2}<h<h_{L}$. This difficulty is resolved by seeking a boundary layer for the inner solution of $(3.2 a b)$ in the neighborhood of $\eta=0$. A different representation of the solution is needed in the neighborhood of $\eta=0$. Consider an inner solution in terms of $z=\eta / \beta$,

$$
h=\hat{h}^{0}(z)+\beta \hat{h}^{1}(z)+O\left(\beta^{2}\right), \quad \Gamma=\hat{\Gamma}^{0}(z)+\beta \hat{\Gamma}^{1}(z)+O\left(\beta^{2}\right) .
$$

Substituting into $(3.2 b)$ yields that $d \Gamma / d z=O(\beta) \rightarrow 0$, hence to leading order $\Gamma$ is 

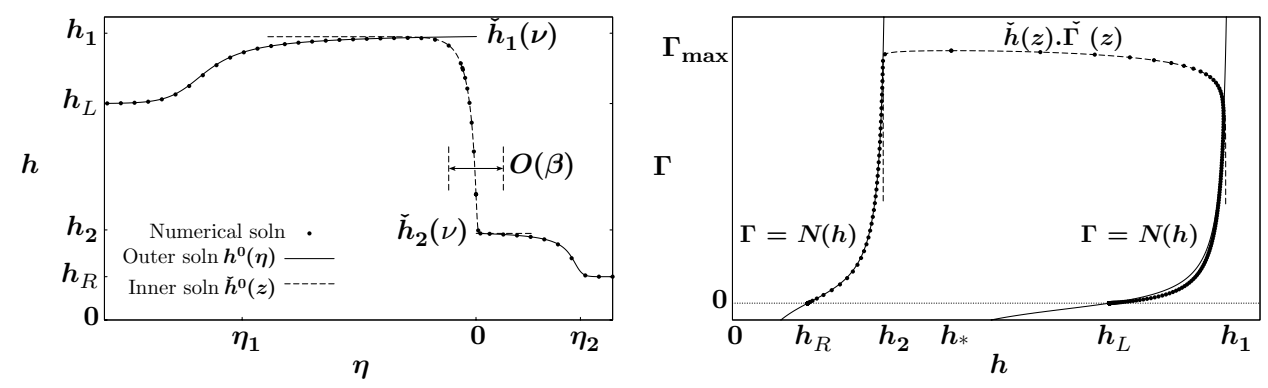

FIGURE 12. Construction of the matched asymptotic solution for $\beta \rightarrow 0$ : (Left) $h(\eta)$ profile from outer solutions $h_{L, R}^{0}(\eta)$ (solid curves) for $|z|>0$ and inner solution $\hat{h}^{0}(z)$ (dashed), and (Right) similarly in the phase plane where the outer solutions are given by the nullcline $\Gamma=N(h)$. The dotted curve corresponds to one of the numerically computed solutions from Figure 11.

constant $; \hat{\Gamma}^{0}(z) \equiv \Gamma_{\max }$. Consequently, the leading order behavior of $h(z)$ is given by

$$
\frac{d \hat{h}^{0}}{d z}=\frac{P_{1}\left(\hat{h}^{0}\right) \Gamma_{\max } \hat{h}^{0}+4 \delta P_{0}\left(\hat{h}^{0}\right)}{\left(\hat{h}^{0}\right)^{3}\left(\Gamma_{\max } \hat{h}^{0}+4 \delta\right)}, \quad \hat{h}^{0}(0)=h_{*}
$$

The terms in the numerator and denominator on the right hand side of this ODE are homogeneously weighted by $\Gamma_{\max }$ and $\delta$. Hence it is convenient to define a ratio of these parameters,

$$
v \equiv \frac{\delta}{\Gamma_{\max }} \geqslant 0,
$$

and re-write (3.28) in terms of this single parameter as

$$
\frac{d \hat{h}^{0}}{d z}=\frac{\bar{P}\left(\hat{h}^{0}\right)}{\left(\hat{h}^{0}\right)^{3}\left(\hat{h}^{0}+4 v\right)}, \quad \bar{P}(h) \equiv h P_{1}(h)+4 v P_{0}(h) .
$$

The equilibria of (3.28) are the roots of $N\left(\hat{h}^{0}\right)=\Gamma_{\max }$, corresponding to the positive zeroes of the fourth-order polynomial $\bar{P}\left(\hat{h}^{0}\right)$. We now give a lemma with the key properties of these zeroes:

Lemma 3.3 For each $v \geqslant 0$, equation (3.30) has exactly two positive equilibria $\bar{h}_{1}(v)$ and $\bar{h}_{2}(v)$ satisfying

$$
\begin{aligned}
& h_{L} \leqslant \bar{h}_{1}(v) \leqslant h_{1}, \quad \bar{h}_{1}(0)=h_{1}, \quad \lim _{v \rightarrow \infty} \bar{h}_{1}(v)=h_{L}, \\
& h_{R} \leqslant \bar{h}_{2}(v) \leqslant h_{2}, \quad \bar{h}_{2}(0)=h_{2}, \quad \lim _{v \rightarrow \infty} \bar{h}_{2}(v)=h_{R} .
\end{aligned}
$$

Proof We make use of Lemma 3.1 for the ordering (3.7) of the zeroes of $P_{0}, P_{1}$. From Figure 6, we see that $\bar{P}\left(h_{L}\right)<0<\bar{P}\left(h_{1}\right)$, so that $\bar{P}(h)$ has a zero $h=\bar{h}_{1}(v)$ between $h_{L}$ and $h_{1}$. Similarly, $\bar{P}\left(h_{2}\right)<0<\bar{P}\left(h_{R}\right)$, so that $\bar{P}(h)$ has a zero $h=\bar{h}_{2}(v)$ in the interval $\left(h_{R}, h_{2}\right)$. 
To prove that $\bar{h}_{1}(v), \bar{h}_{2}(v)$ are the only two positive zeroes for each $v>0$, we show that the fourth degree polynomial $\bar{P}(h)$ has two negative zeroes. At $h_{M}=-\left(h_{L}+h_{R}\right)$, $P_{0}\left(h_{M}\right)=0$, see $(3.5)$, so $\bar{P}\left(h_{M}\right)=h_{M} P_{1}\left(h_{M}\right)$. From Lemma $3.1, h_{L}+h_{R}<h_{1}+h_{2}$, so from (3.6) we conclude that $\bar{P}\left(h_{M}\right)<0$. Further, note that $\bar{P}(0)=v \bar{P}_{0}(0)>0$, and $\bar{P}(h) \sim h^{4} \rightarrow+\infty$ as $h \rightarrow-\infty$. Consequently, $\bar{P}(h)$ changes sign precisely twice along the negative $h$ axis.

Finally, in the limit $v \rightarrow 0$ we have $\bar{P}(h) \sim h P_{1}(h)$ with zeroes $h_{1}, h_{2}$, and for $v \rightarrow \infty$ the polynomial reduces to $\bar{P}(h) \sim v P_{0}(h)$ with zeroes $h_{L}, h_{R}$.

Noting that equation (3.30) is a first-order ODE, and since $d \hat{h}^{0} / d z<0$ at $z=0$ (i.e., $\bar{P}\left(h_{*}\right)<0$ ), the solution $\hat{h}^{0}(z)$ is monotone decreasing for all $z$ with limiting behaviors

$$
\hat{h}^{0}(z \rightarrow-\infty) \rightarrow \bar{h}_{1}(v), \quad \hat{h}^{0}(z \rightarrow \infty) \rightarrow \bar{h}_{2}(v) .
$$

In terms of matched asymptotics, these far-field limits of the inner solution give the initial conditions for the two portions of the leading order outer solution (3.25) on $\eta<0$ and $\eta>0$

$$
h^{0}\left(0^{-}\right)=\bar{h}_{1}(v), \quad h^{0}\left(0^{+}\right)=\bar{h}_{2}(v), \quad \Gamma^{0}(0)=\Gamma_{\max } .
$$

In the limit of large $\Gamma_{\max }$, we can express these zeroes in terms of perturbation expansions for $v \rightarrow 0$,

$$
\bar{h}_{1}(v)=h_{1}-v \frac{4 P_{0}\left(h_{1}\right)}{h_{1} P_{1}^{\prime}\left(h_{1}\right)}-v^{2} \frac{8 P_{0}\left(h_{1}\right)}{h_{1}^{2} P_{1}^{\prime}\left(h_{1}\right)^{2}}\left(P_{0}\left(h_{1}\right) \frac{P_{1}^{\prime \prime}\left(h_{1}\right)}{P_{1}^{\prime}\left(h_{1}\right)}+\frac{2 P_{0}\left(h_{1}\right)}{h_{1}}-2 P_{0}^{\prime}\left(h_{1}\right)\right)+O\left(v^{3}\right),
$$

and similarly for $\bar{h}_{2}(v)$ with all $h_{1}$ replaced by $h_{2}$ above. We note that (3.33) agrees with the expansion for $\breve{h}\left(0^{ \pm}\right)$given in Section 3.2 up to $O\left(\Gamma_{\max }^{-1}\right)$. The differences between the limits $\Gamma_{\max } \rightarrow \infty$ with $\beta$ fixed versus $\beta \rightarrow 0$ with $\Gamma_{\max }$ fixed enter at $O\left(\Gamma_{\max }^{-2}\right)$ in these expansions.

Conditions (3.32) are consistent with the description of the solution executing a fast jump at $\Gamma=\Gamma_{\max }$ between the two branches of the slow manifold (the $\Gamma=N(h)$ nullcline). More details on the structure of $\Gamma$ in the inner solution are obtained by going to next order (see Figure 12),

$$
\frac{d \hat{\Gamma}^{1}}{d z}=\frac{2 s\left(\hat{h}^{0}-h_{*}\right)}{\hat{h}^{0}\left(\hat{h}^{0}+4 v\right)}, \quad \hat{\Gamma}^{1}(0)=0,
$$

which is included in Figure 12(right).

For completeness we also consider the $v \rightarrow \infty$ limit of (3.30) which to leading order yields

$$
\frac{d \hat{h}^{0}}{d z}=\frac{P_{0}\left(\hat{h}^{0}\right)}{\left(\hat{h}^{0}\right)^{3}}
$$

As expected from Lemma 3.3, this equation describes an inner layer with a transition from $h_{L}$ to $h_{R}$. Expressing $v \rightarrow \infty$ in terms of our original parameters determines the 

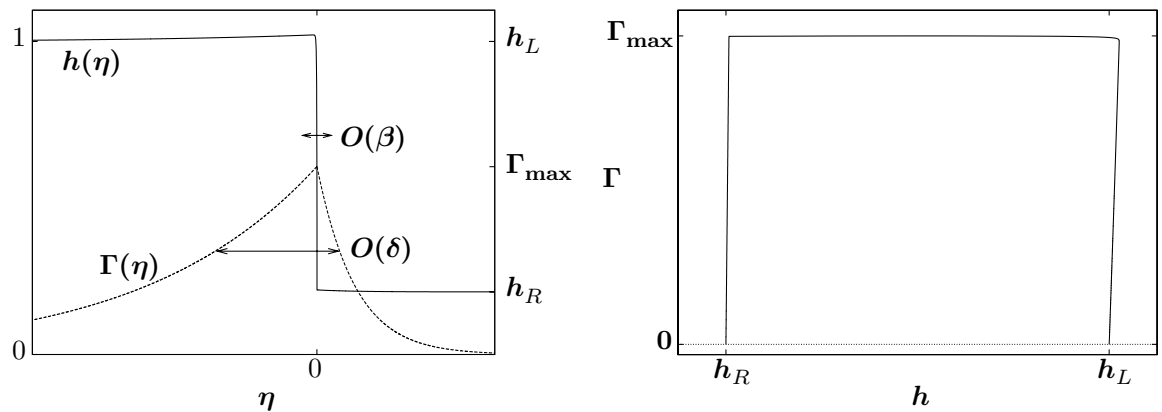

FiguRE 13. Solutions for $\beta \rightarrow 0$ and $v \rightarrow \infty$ : (Left) nested boundary layers for $\beta \rightarrow 0$ and $\delta \rightarrow \infty$ and (Right) the phase plane representation approaches a three-segment polygonal curve.

relations $\beta \ll \Gamma_{\max } \ll \delta$, where we take $\Gamma_{\max }=O(1)$. A consequence of this is that $\Gamma$ is well approximated by the linearized behaviors $(3.8,3.9)$,

$$
\Gamma(\eta) \sim \Gamma_{\max } \begin{cases}\exp \left(\lambda_{2}^{L} \eta\right) & \eta<0 \\ \exp \left(\lambda_{2}^{R} \eta\right) & \eta>0\end{cases}
$$

where the exponential decay rates have $\lambda_{2}=O\left(\delta^{-1}\right)$. Thus in this limit the solution exhibits nested boundary layers at $\eta=0$ : the jump in $h$ occurs on the narrow $O(\beta)$ layer, which is nested within the wider $O(\delta)$ layer on which $\Gamma$ decays to zero, see Figure 13 .

We conclude by pointing out the equivalence of the limiting values of the $\beta \rightarrow 0$ leading order outer solution, $\left(h^{0}(\eta), \Gamma^{0}(\eta)\right)$ at $\eta \rightarrow 0^{ \pm}$, with the jump conditions at $\eta=0$ for the $\beta=0$ weak solution with $\delta \geqslant 0$,

$$
{ }_{-S}[h]-\frac{1}{2}\left[h^{2} \Gamma^{\prime}\right]+\frac{1}{3}\left[h^{3}\right]=0, \quad-\Gamma_{\max }\left[h \Gamma^{\prime}\right]+\frac{1}{2} \Gamma_{\max }\left[h^{2}\right]=\delta\left[\Gamma^{\prime}\right] .
$$

If $\delta>0$, the jump in $h, \Gamma^{\prime}$ at $\eta=0$ is the only discontinuity. Recall from (1.7) that the surface fluid speed is given by $u_{h}=\frac{1}{2} h^{2}-h \Gamma_{x}$. Consequently, the second jump condition in (3.37) is

$$
\left[u_{h}\right]=v\left[\Gamma^{\prime}\right]<0 .
$$

That is, the spreading associated with diffusion of surfactant on the surface is balanced by the transport of surfactant toward the discontinuity at $\eta=0$. Moreover, we observe that this discontinuity is compressive in the sense that the surface velocity, $u_{h}(\eta)$ satisfies

$$
u_{h}\left(0^{+}\right)<s<u_{h}\left(0^{-}\right)
$$

Next, we examine what occurs in the limit $\delta \rightarrow 0$.

\subsection{Solution for the $\delta \rightarrow 0$ limit}

From Figure 14 we see that the limit $\delta \rightarrow 0$ manifests a dramatic change in the structure of the solution near $\eta_{1}$ and $\eta_{2}$. While the traveling wave again appears to approach the weak solution of Section 2, we will see that the behavior near the discontinuities takes a significantly different form than in Section 3.3. 

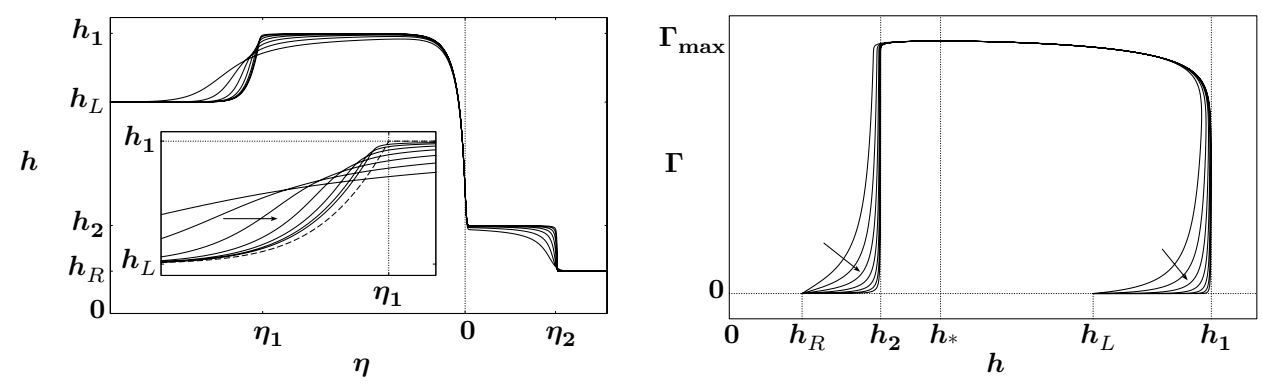

FIGURE 14. Trends for $\delta \rightarrow 0$ : (left) Notable changes occur in the numerically computed solution profiles at $\eta=\eta_{1}, \eta_{2}$, i.e. where $\Gamma \searrow 0$. The inset shows a close-up of the corner layer at $\eta_{1}$ for $\delta \rightarrow 0$. (Right) The solutions for $\delta \rightarrow 0$ shown in the $(h, \Gamma)$ phase plane. See Figure 15 for corresponding $\Gamma(\eta)$ profiles.

We begin with a regular expansion for the outer solution

$$
h=h^{0}(\eta)+\delta h^{1}(\eta)+O\left(\delta^{2}\right), \quad \Gamma=\Gamma^{0}(\eta)+\delta \Gamma^{1}(\eta)+O\left(\delta^{2}\right) .
$$

For $\Gamma>0,(3.2 a b)$ yield the leading order equations

$$
\frac{d h^{0}}{d \eta}=\frac{P_{1}\left(h^{0}\right)}{\beta\left(h^{0}\right)^{3}}, \quad \frac{d \Gamma^{0}}{d \eta}=\frac{2 s\left(h^{0}-h_{*}\right)}{\left(h^{0}\right)^{2}},
$$

with initial conditions $h^{0}(0)=h_{*}, \Gamma^{0}(0)=\Gamma_{\max }$. We consider the form of the solution for $\eta \leqslant 0$; results for $\eta \geqslant 0$ follow analogously.

The domain of applicability of (3.39) is limited by the consideration that the surfactant concentration must be non-negative. For $\delta=0$, we define the edge of the region of support of the leading order solution by $\bar{\eta}_{1}$, where $\Gamma^{0}\left(\bar{\eta}_{1}\right)=0$. This finite position can be obtained by numerical integration of (3.39). Scaling arguments show that

$$
\bar{\eta}_{1}=-\frac{\Gamma_{\max }}{g_{1}\left(\Gamma_{\max } / \beta\right)},
$$

where $g_{1}(\mu)$ is numerically observed to be a monotone increasing function and has $g_{1}(\mu \rightarrow \infty) \rightarrow G_{1}$, consistent with $(2.7) . \Gamma^{0}(\eta)$ hits zero at $\bar{\eta}_{1}$ with finite slope and for $\eta<\bar{\eta}_{1}$, we take the non-negative truncation, $\Gamma^{0}(\eta) \equiv 0$. That is, $\Gamma^{0}$ has a corner at $\bar{\eta}_{1}$, see Figure 15(left); we will show that $h^{0}(\eta)$ also has a corner there, see Figure 14(left). The influence of finite $\delta$ is to regularize this behavior; for $\delta>0, d \Gamma / d \eta$ can no longer jump and hence $\Gamma$ will lose its compact support, see Figure 15(left). To describe this behavior, we make use of the asymptotic analysis developed by J. R. King [12, 13] for the solutions of a regularized porous medium equation.

The analysis begins with the examination of the local structure of the outer solution of $h(\eta), \Gamma(\eta)$ for $\eta \rightarrow \bar{\eta}_{1}$ with $\eta \geqslant \bar{\eta}_{1}$. To leading order, (3.39) yields

$$
h^{0}(\eta) \sim c_{0} \quad \Gamma^{0}(\eta) \sim b_{1}\left(\eta-\bar{\eta}_{1}\right) \quad \text { as } \eta \rightarrow \bar{\eta}_{1}^{+},
$$

where $b_{1}=2 s\left(c_{0}-h_{*}\right) / c_{0}^{2}$ and the positive constant $c_{0}$ can be obtained numerically by integrating (3.39) from $\eta=0$. From the results of previous sections, if $\beta \rightarrow 0$ or $\Gamma_{\max } \rightarrow \infty$, 

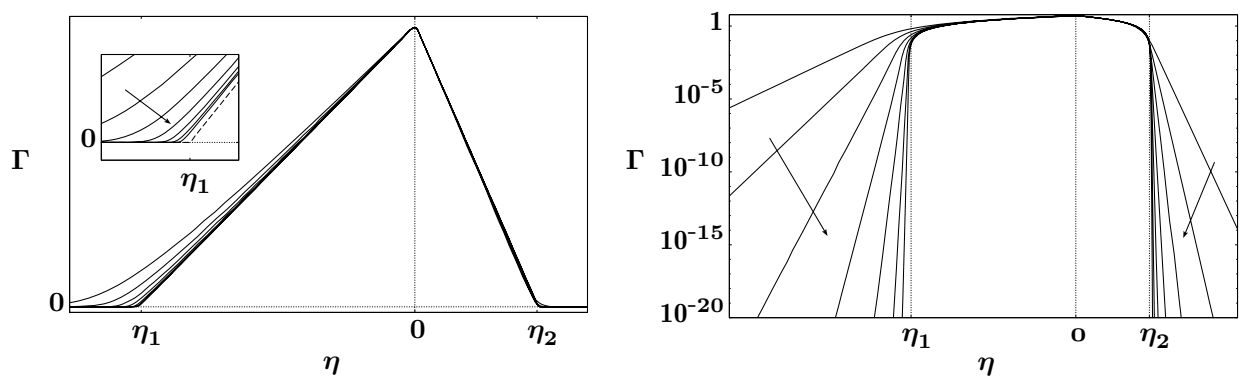

Figure 15. (Left) Numerically computed $\Gamma(\eta)$ profiles for $\delta \rightarrow 0$ (corresponding to the $h$ profiles from Figure 14). The inset shows a close-up of the corner layer at $\eta_{1}$. (Right) The $\Gamma$ profiles in a semi-log plot showing convergence to a compactly-supported weak solution as exponentially small terms decay outside the interval $\bar{\eta}_{1}<\eta<\bar{\eta}_{2}$.

we expect $c_{0} \rightarrow h_{1}$ and $b_{1} \rightarrow G_{1}$. For convenience, we will assume these limiting values apply (using the $\Gamma_{\max } \rightarrow \infty$ limit). This simplifies some equations while retaining the same qualitative form of the local structure of the solution that would hold for any finite $\Gamma_{\max }$. Hence we can proceed without significant loss of generality in taking $c_{0}=h_{1}$ and $b_{1}=G_{1}$.

Proceeding to $O(\delta)$, letting $\zeta=\eta-\bar{\eta}_{1}$, the local form of the next order equations is

$$
\frac{d h^{1}}{d \zeta} \sim \frac{P_{1}^{\prime}\left(h_{1}\right)}{\beta h_{1}^{3}} h^{1}(\zeta)+\frac{4 P_{0}\left(h_{1}\right)}{\beta h_{1}^{4} G_{1} \zeta}, \quad \frac{d \Gamma^{1}}{d \zeta} \sim-\frac{2 s\left(h_{1}-2 h_{*}\right)}{h_{1}^{3}} h^{1}(\zeta)-\frac{8 s\left(h_{1}-h_{*}\right)}{h_{1}^{3} G_{1} \zeta} .
$$

Solving these equations for $\zeta \rightarrow 0$ yields

$$
h^{1}(\zeta) \sim \frac{4 P_{0}\left(h_{1}\right)}{\beta h_{1}^{4} G_{1}} \ln \zeta+c_{1}, \quad \Gamma^{1}(\zeta) \sim-\frac{8 s\left(h_{1}-h_{*}\right)}{h_{1}^{3} G_{1}} \ln \zeta+b_{2},
$$

where $c_{1}, b_{2}$ are constants determined by the initial conditions on $h, \Gamma$. These solutions are unbounded as $\zeta \rightarrow 0$, showing the nonuniformity of (3.38) and the need for interior boundary layers.

King showed that it is convenient to define the location of the interior layer as where $\Gamma=\delta$, i.e.

$$
\Gamma(\eta)=\delta \quad \text { at } \eta=\ell(\delta)
$$

where $\ell \rightarrow \bar{\eta}_{1}$ as $\delta \rightarrow 0$. Next, we write scaled solutions as

$$
\Gamma=\delta \hat{\Gamma}(\xi) \quad h=h_{1}+\delta \hat{h}(\xi) \quad \eta=\ell+\delta \xi,
$$

which to leading order $\left(\hat{\Gamma} \sim \hat{\Gamma}^{0}+O(\delta), \hat{h} \sim \hat{h}^{0}+O(\delta)\right)$ satisfy

$$
\frac{d \hat{h}^{0}}{d \xi}=\frac{4 P_{0}\left(h_{1}\right)}{\beta h_{1}^{3}\left(\hat{\Gamma}^{0} h_{1}+4\right)}, \quad \frac{d \hat{\Gamma}^{0}}{d \xi}=\frac{2 s\left(h_{1}-h_{*}\right) \hat{\Gamma}^{0}}{h_{1}\left(\hat{\Gamma}^{0} h_{1}+4\right)} .
$$

Integrating the $\hat{\Gamma}^{0}$ equation and applying the boundary condition $\hat{\Gamma}(0)=1$, we obtain 
the implicit relation

$$
h_{1}^{2} \hat{\Gamma}^{0}+4 h_{1} \ln \hat{\Gamma}^{0}=2 s\left(h_{1}-h_{*}\right) \xi+h_{1}^{2} .
$$

From this we can obtain the limiting behavior for $\hat{\Gamma}^{0} \rightarrow \infty$,

$$
\hat{\Gamma}^{0}(\xi) \sim \frac{2 s\left(h_{1}-h_{*}\right)}{h_{1}^{2}} \xi-\frac{4}{h_{1}} \ln \left(\frac{2 s\left(h_{1}-h_{*}\right)}{h_{1}^{2}} \xi\right)+1 \quad \text { as } \xi \rightarrow \infty .
$$

We then seek the form of the layer position in terms of the expansion

$$
\ell(\delta) \sim \bar{\eta}_{1}+\bar{\eta}_{a} \delta \ln \delta+\bar{\eta}_{b} \delta, \quad \delta \rightarrow 0,
$$

where coefficients $\bar{\eta}_{a}, \bar{\eta}_{b}$ are to be determined by matching with the outer solution. Expanding the outer solution in terms of $\xi$ for $\ln \delta \ll \xi \ll 1 / \delta$ yields

$$
\frac{\Gamma^{0}(\xi)+\delta \Gamma^{1}(\xi)}{\delta} \sim \frac{2 s\left(h_{1}-h_{*}\right)}{h_{1}^{2}} \xi+\frac{2 s\left(h_{1}-h_{*}\right)}{h_{1}^{2}} \bar{\eta}_{a} \ln \delta+\frac{2 s\left(h_{1}-h_{*}\right)}{h_{1}^{2}} \bar{\eta}_{b}-\frac{8 s\left(h_{1}-h_{*}\right)}{h_{1}^{3} G_{1}} \ln (\delta \xi) .
$$

We proceed to match (3.48) to (3.50) term by term. At $O(\ln \delta)$ and $O(1)$ respectively we determine that

$$
\bar{\eta}_{a}=\frac{8 s\left(h_{1}-h_{*}\right)}{h_{1}^{3} G_{1}^{2}}, \quad \bar{\eta}_{b}=\frac{1}{G_{1}}\left(1-\frac{4}{h_{1}} \ln \left[\frac{2 s\left(h_{1}-h_{*}\right)}{h_{1}^{2}}\right]\right) .
$$

Returning to (3.47), we find the limiting behavior for $\hat{\Gamma}^{0} \rightarrow 0$ is given by

$$
\hat{\Gamma}^{0}(\xi) \sim \exp \left(\frac{1}{4} h_{1}+\frac{s\left(h_{1}-h_{*}\right)}{2 h_{1}} \xi\right), \quad \text { as } \xi \rightarrow-\infty ;
$$

this behavior must be matched to the outer solution for $|\eta| \gg \ell$ with $\Gamma=o(\delta)$,

$$
\frac{d h}{d \eta}=\frac{P_{0}(h)}{\beta h^{3}}, \quad \frac{d \Gamma}{d \eta}=\frac{s\left(h-h_{*}\right) \Gamma}{2 \delta h} .
$$

These equations describe the form of $\Gamma(\eta)$ that connects (3.52) to the results from linear stability (3.15), as $h$ transitions from $h \sim h_{1}$ to $h \rightarrow h_{L}$. Observe that after rescaling $\xi$, (3.52) agrees with $\Gamma=O\left(e^{\hat{\lambda}_{2} \eta}\right)$ with $\hat{\lambda}_{2}=s\left(h_{1}-h_{*}\right) /\left(2 h_{1} \delta\right)$, and like $\lambda_{2}$ in $(3.8), \hat{\lambda}_{2}=O\left(\delta^{-1}\right)$ as $\delta \rightarrow 0$. We can conclude that $\Gamma$ is exponentially small for all $|\eta| \gg \ell$. These results clarify how the compactly-supported weak solution for $\Gamma(\eta)$ is approached as $\delta \rightarrow 0$. For $\delta=0, \Gamma$ has a corner at $\bar{\eta}_{1}$ with the jump in the slope being $\left[\Gamma^{\prime}\right] \approx G_{1}-0$. The slope $h^{\prime}(\eta)$ also approaches a jump discontinuity (i.e., a corner for $h$ ) with $\left[h^{\prime}\right] \approx 0-P_{0}\left(h_{1}\right) /\left(\beta h_{1}^{3}\right)$. The leading order outer solution from $(3.39,3.53)$ can be interpreted as coming from a piecewise-defined dynamical system:

$$
\frac{d h}{d \eta}=\left\{\begin{array}{ll}
P_{1}(h) / \beta h^{3}, & \Gamma>0 \\
P_{0}(h) / \beta h^{3}, & \Gamma=0
\end{array} \quad \frac{d \Gamma}{d \eta}= \begin{cases}2 s\left(h-h_{*}\right) / h^{2}, & \Gamma>0 \\
0, & \Gamma=0 .\end{cases}\right.
$$


In this system, the polynomials $P_{0}, P_{1}$ continue to play separate roles when $\delta=0$. Derivatives of both $h$ and $\Gamma$ jump at $\Gamma=0$. Initial conditions with $\Gamma>0$ lead to well-defined compactly-supported solutions, parametrized by $\Gamma_{\max }$, as in Theorem 3.2.

\subsection{Overview of the second-order problem}

We have shown that the second-order regularizations in $\beta$ and $\delta$, while both taking the forms of singular perturbations, do not dramatically change the global structure of the traveling wave solutions. As in most singular perturbations problems, corrections are introduced in boundary layers, at $\eta=0$ or $\eta=\eta_{1}, \eta_{2}$ respectively, but these alterations do not change the algebraic relations determined by the far-field boundary conditions that define the overall form of the limiting weak traveling wave solution (2.5).

The analysis in this section can be put into a single framework with the rescaling

$$
\Gamma=\delta \hat{\Gamma}(z), \quad h=\hat{h}(z), \quad \eta=\delta z,
$$

yielding the system

$$
\rho \frac{d \hat{h}}{d z}=\frac{P_{1}(\hat{h}) \hat{\Gamma} \hat{h}+4 P_{0}(\hat{h})}{\hat{h}^{3}(\hat{\Gamma} \hat{h}+4)}, \quad \frac{d \hat{\Gamma}}{d z}=\frac{2 s \hat{\Gamma}\left(\hat{h}-h_{*}\right)}{\hat{h}(\hat{\Gamma} \hat{h}+4)},
$$

where $\rho=\beta / \delta$. The initial conditions (3.12) then take the form $\hat{h}(0)=h_{*}, \hat{\Gamma}(0)=v^{-1}$, where $v=\delta / \Gamma_{\max }$, recall (3.29). The traveling wave solutions $\hat{h}(z), \hat{\Gamma}(z)$ now explicitly depend only on two parameters, $v$ and the ratio $\rho$. The limit $\Gamma_{\max } \rightarrow \infty$ examined in Section 3.2 is given by $v \rightarrow 0$. The limit $\beta \rightarrow 0$ examined in Section 3.3 is given by $\rho \rightarrow 0$. The behaviour for $\beta \rightarrow 0$ with $v \rightarrow \infty$ was also considered in that section. The limit $\delta \rightarrow 0$ examined in Section 3.4 corresponds to $\rho \rightarrow \infty$ with $v \rightarrow 0$. All of these limits and the boundary between monotone and non-monotone $h(\eta)$ solutions, $v^{\text {crit }}(\rho)=\delta / \Gamma_{\max }^{\text {crit }}(\rho)$, are shown in Figure 16. As described in Section 3.1, all solutions with $\rho<\rho_{L}$ are nonmonotone, see (3.16). For $\rho \rightarrow \infty$, $v^{\text {crit }}=O\left(\rho^{-1}\right)$, identifying a distinguished limit similar to $(3.24)$.

\section{Traveling waves with fourth-order regularization}

In this section, we return to the full system including capillary effects (1.9):

$$
\begin{gathered}
-s h+\frac{1}{3} h^{3}-\frac{1}{2} h^{2} \frac{d \Gamma}{d \eta}=\frac{1}{3} \beta h^{3} \frac{d h}{d \eta}-\frac{1}{3} \kappa h^{3} \frac{d^{3} h}{d \eta^{3}}-k_{h}, \\
-s \Gamma+\frac{1}{2} h^{2} \Gamma-h \Gamma \frac{d \Gamma}{d \eta}=\frac{1}{2} \beta h^{2} \Gamma \frac{d h}{d \eta}-\frac{1}{2} \kappa h^{2} \Gamma \frac{d^{3} h}{d \eta^{3}}+\delta \frac{d \Gamma}{d \eta},
\end{gathered}
$$

These equations can be rearranged into a fourth-order autonomous system,

$$
\kappa \frac{d^{3} h}{d \eta^{3}}=\beta \frac{d h}{d \eta}-\frac{P_{1}(h) \Gamma h+4 \delta P_{0}(h)}{h^{3}(\Gamma h+4 \delta)}, \quad \frac{d \Gamma}{d \eta}=\frac{2 s \Gamma\left(h-h_{*}\right)}{h(\Gamma h+4 \delta)},
$$

where setting $\kappa=0$ recovers the system of Section 3. Numerical simulations of the full PDE system (1.4) suggest that traveling wave solutions satisfying (4.2) exist and are stable 


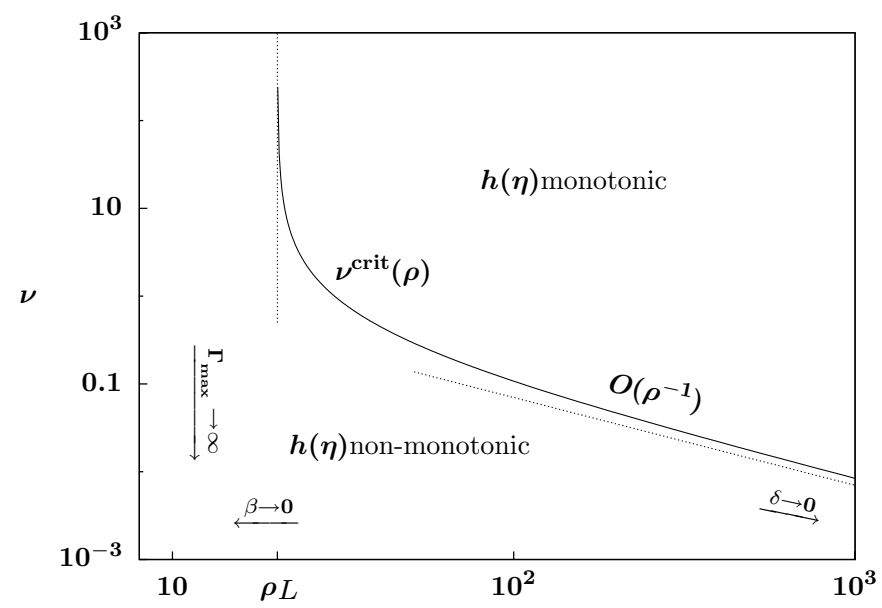

FIGURE 16. The various limits of the second-order system considered in the subsections of Section 3 as represented in the $(\rho, v)$ parameter plane.
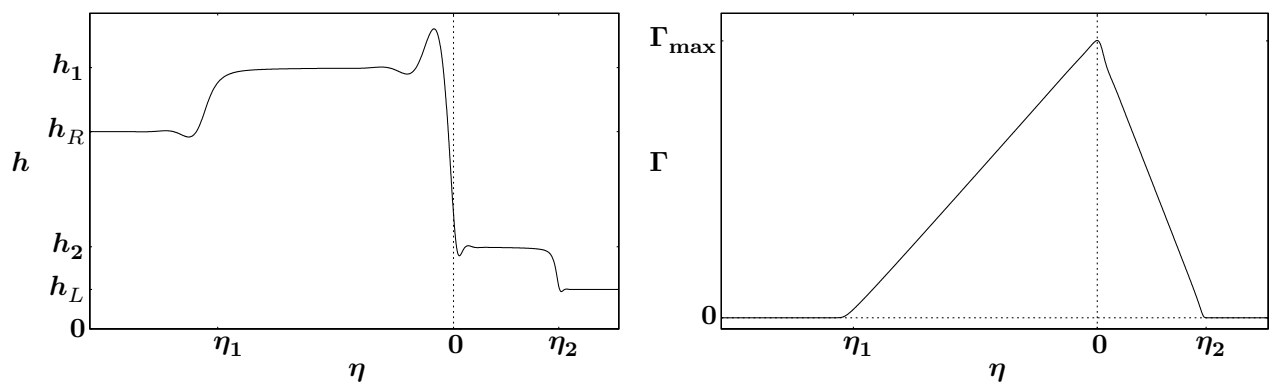

FIGURE 17. The effect of capillarity: numerical solutions with $\kappa>0$, and $\beta, \delta$ both small.

in one dimension, see Figure 17. In contrast to analysis of the second-order model in Section 3, full consideration of the existence of weak traveling wave solutions for this fourth-order system $(4.2 a b)$ is more difficult, and parts will be delayed to further work. In this section, we provide partial results on traveling waves for a reduced version of (4.2) using numerical solutions together with asymptotics, extending the analysis of Section 3. In the absence of surfactant, the balance of the competing $\beta, \kappa$ regularizations in (1.2) determines whether the film height profile will take the form of a monotone front or a capillary ridge $[3,21]$. The monotone decreasing front is analogous to the low-surfactant case, where $\Gamma_{\max }<\Gamma_{\max }^{\text {crit }}(\beta / \delta)$, considered in Section 3.1. To focus attention on the influence of $\kappa$, we take $\beta=0$; this can be interpreted as choosing to study the capillary ridge in the flow down a vertical wall [19]. With $\beta=0$, we avoid complications associated with solutions for positive $\beta$ describing flows down inclined planes with small to finite angles of inclination (i.e., relatively large $\beta$ ) [21]. Thus for the remainder of this section, 
we consider the system

$$
\kappa \frac{d^{3} h}{d \eta^{3}}=-\frac{P_{1}(h) \Gamma h+4 \delta P_{0}(h)}{h^{3}(\Gamma h+4 \delta)}, \quad \frac{d \Gamma}{d \eta}=\frac{2 s \Gamma\left(h-h_{*}\right)}{h(\Gamma h+4 \delta)} .
$$

This system has equilibrium points at $(h, \Gamma)=\left(h_{L}, 0\right)$ and $\left(h_{R}, 0\right)$. Linearizing about $h_{\mathrm{eq}}=\left\{h_{L}, h_{R}\right\}, \Gamma=0$ yields

$$
\frac{d^{3} \tilde{h}}{d \eta^{3}}=-\frac{P_{0}^{\prime}\left(h_{\mathrm{eq}}\right)}{\kappa h_{\mathrm{eq}}^{3}} \tilde{h}-\frac{P_{1}\left(h_{\mathrm{eq}}\right)}{4 \kappa \delta h_{\mathrm{eq}}^{2}} \tilde{\Gamma}, \quad \frac{d \tilde{\Gamma}}{d \eta}=\frac{s\left(h_{\mathrm{eq}}-h_{*}\right)}{2 \delta h_{\mathrm{eq}}} \tilde{\Gamma} .
$$

The equilibrium point $\left(h_{L}, 0\right)$ has eigenvalues

$$
\lambda_{k}=-\omega^{k}\left(\frac{\left(h_{L}-h_{R}\right)\left(2 h_{L}+h_{R}\right)}{\kappa h_{L}^{3}}\right)^{1 / 3}, \quad \lambda_{3}=\frac{s\left(h_{L}-h_{*}\right)}{2 \delta h_{L}},
$$

where $k=0,1,2$ and $\omega=e^{i 2 \pi / 3}$. That is, it has a single stable direction with $\lambda_{0}<0$ and has a three-dimensional unstable manifold $W_{L}^{U}$ with $\operatorname{Re}\left(\lambda_{1,2}\right)>0$ and $\lambda_{3}>0$. Similarly, $\left(h_{R}, 0\right)$ has eigenvalues

$$
\lambda_{k}=\omega^{k}\left(\frac{\left(h_{L}-h_{R}\right)\left(2 h_{R}+h_{L}\right)}{\kappa h_{L}^{3}}\right)^{1 / 3}, \quad \lambda_{3}=\frac{s\left(h_{R}-h_{*}\right)}{2 \delta h_{R}} ;
$$

it has a three-dimensional stable manifold $W_{R}^{S}\left(\operatorname{Re}\left(\lambda_{1,2}\right)<0\right.$ and $\left.\lambda_{3}<0\right)$ and a single unstable direction with $\lambda_{0}>0$. In particular, as $|\eta| \rightarrow \infty, h(\eta)$ spirals into the equilibria with decay rates $O\left(\kappa^{-1 / 3}\right)$ while $\Gamma(\eta)$ exhibits monotone exponential behavior with rate $O\left(\delta^{-1}\right)$. As in the second-order problem, the linearized structure of $\Gamma(\eta)$ is given by a single eigenmode (here $\lambda_{3}$ ) while the form of $h(\eta)$ depends on the relative sizes of $\operatorname{Re}\left(\lambda_{1,2}\right)$ vs. $\lambda_{3}$ as in (3.16). Heteroclinic orbits connecting equilibria lie in the two-dimensional intersection of the three-dimensional manifolds, $W_{L}^{U}$ and $W_{R}^{S}$. Consequently, we expect a one-parameter family of solutions, as in Section 3. These features are seen clearly in Figures 17 and 18, plots of numerical solutions of the PDE system for $\kappa>0$ and $\beta>0, \delta>0$ both taken to be small, and nonzero, in order to stabilize the numerical method. In Figure 17, we show graphs of $h$ and $\Gamma$, in which oscillations of $h$ can be observed. In Figure 18, we show two projections of the four-dimensional $\left(h, h_{x}, h_{x x}, \Gamma\right)$ phase portrait, with data obtained through finite differences of the numerical PDE solution of Figure 17. In the left hand plots of Figures 17 and 18, it is possible to follow the trajectory from $h_{L}$ to $h_{R}$, relating the oscillations in the graph of $h$ to the spirals in the phase portrait. In the next subsection, we explain the structure of the phase portraits in more detail using asymptotics.

\subsection{Solutions for $\kappa \rightarrow 0$}

In studying the structure of traveling wave solutions of (4.3) for $\kappa \rightarrow 0$, we can take advantage of similarities to our analysis of (3.2) in Section 3.3. In particular, the leading order outer solution for (4.3) is the same as equation (3.25) in Section 3.3. The outer solution consequently consists of smooth monotonic functions for $h, \Gamma$, with $h$ connecting 

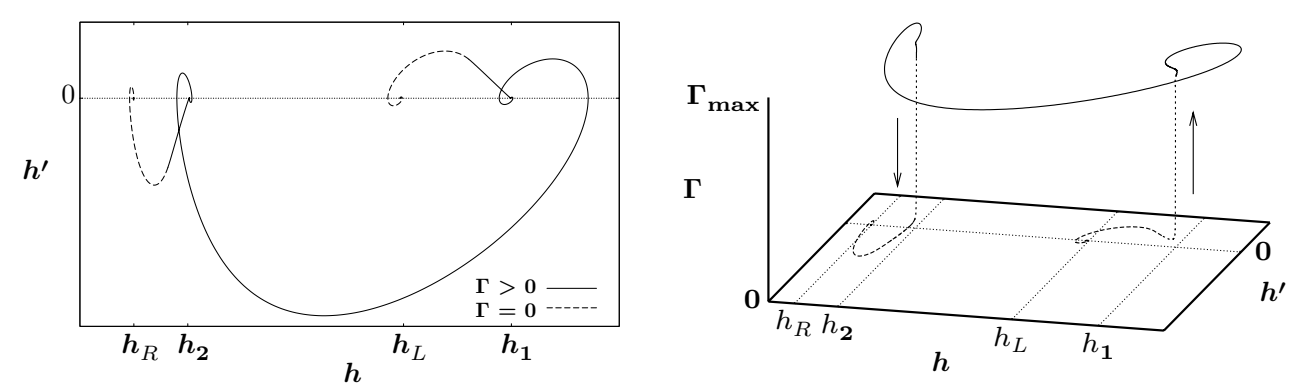

FIGURE 18. Phase plots of numerical solutions with $\kappa>0$, and $\beta, \delta$ both small. (Left) $\left(h, h^{\prime}\right)$. (Right) $\left(h, h^{\prime}, \Gamma\right)$. Line styles indicate relative sizes of $\Gamma$ in the different sections of the curves. The $O\left(\Gamma_{\max }\right)$ section is the inner solution, cf., equation (4.8).

$h_{L}$ to $\bar{h}_{1}(v)$ and $h_{R}$ to $\bar{h}_{2}(v)$ (recall that $v=\delta / \Gamma_{\max }$ ) for $\eta$ away from $\eta=0$. Due to the presence of complex eigenvalues for system (4.4), these solutions have weak oscillatory structure that enters at $O\left(\kappa^{1 / 3}\right)$.

To describe the inner solution, a boundary layer at $\eta=0$, we scale $\eta$ as $\eta=z / \kappa^{1 / 3}$. Then, with $\hat{h}(z)=h(\eta)$ and $\hat{\Gamma}(z)=\Gamma(\eta)$, we have

$$
\frac{d^{3} \hat{h}}{d z^{3}}=-\frac{P_{1}(\hat{h}) \hat{\Gamma} \hat{h}+4 \delta P_{0}(\hat{h})}{\hat{h}^{3}(\hat{\Gamma} \hat{h}+4 \delta)}, \quad \frac{d \hat{\Gamma}}{d z}=\kappa^{1 / 3} \frac{2 s \hat{\Gamma}\left(\hat{h}-h_{*}\right)}{\hat{h}(\hat{\Gamma} \hat{h}+4 \delta)} .
$$

As in the second-order problem, we seek the solution satisfying initial conditions $\hat{\Gamma}(0)=$ $\Gamma_{\max }$ and $\hat{h}(0)=h_{*}$. However, for the current problem, the additional initial conditions for $\hat{h}^{\prime}(0)$ and $\hat{h}^{\prime \prime}(0)$ are not obvious. Writing $\hat{h}, \hat{\Gamma}$ as regular perturbation expansions in powers of $\kappa^{1 / 3}$ and keeping only leading order terms for $\kappa \rightarrow 0, \hat{h} \sim \hat{h}^{0}, \hat{\Gamma} \sim \hat{\Gamma}^{0}$, we observe that the leading order surfactant concentration is constant across the boundary layer, $\hat{\Gamma}^{0}(z) \equiv \Gamma_{\max }$. Comparable to $(3.30), \hat{h}^{0}(z)$ satisfies

$$
\frac{d^{3} \hat{h}^{0}}{d z^{3}}=-\frac{\bar{P}\left(\hat{h}^{0}\right)}{\left(\hat{h}^{0}\right)^{3}\left(\hat{h}^{0}+4 v\right)},
$$

where $\bar{P}(h)$ and $v$ are defined as before. Equation (4.8) is of the general form

$$
\frac{d^{3} h}{d z^{3}}=G(h) .
$$

Nonlinear third-order differential equations of this form have been the subject of numerous studies, many motivated by different forms of thin film flows [1, 2, 8, 28, 29]. As in (3.30), this system has the equilibria $\bar{h}_{1}$ and $\bar{h}_{2}$. A theorem by Mock [18] establishes the existence of a heteroclinic orbit from $\bar{h}_{1}$ to $\bar{h}_{2}$ under some simple assumptions on $G(h)$. Linearizing about the equilibria yields the equation $\tilde{h}^{\prime \prime \prime}=G^{\prime}(\bar{h}) \tilde{h}$ with eigenvalues given by $\lambda^{3}=G^{\prime}(\bar{h})$. At $\bar{h}_{1}$, since $G^{\prime}\left(\bar{h}_{1}\right)<0$ so there is one negative and a complex conjugate pair of eigenvalues with $\operatorname{Re}\left(\lambda_{1,2}\right)>0$. Consequently, there is a two-dimensional unstable manifold and a onedimensional stable manifold associated with $\bar{h}_{1}$. Similarly, since $G^{\prime}\left(\bar{h}_{2}\right)>0$, there is a two-dimensional stable manifold and a one-dimensional unstable manifold associated 

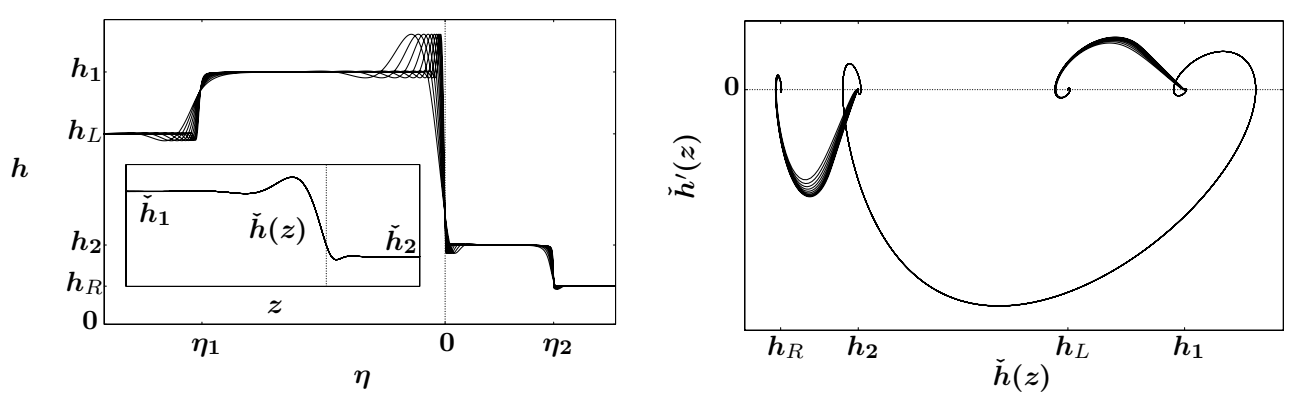

Figure 19. (Left) Height profiles for $\kappa \rightarrow 0$. Here $h_{L}=1.0, h_{R}=0.2, \beta=0, \delta=4 e-4, \Gamma_{\max }=3$, and $\kappa=2^{-n} \times 10^{-1}$ for $n=0,1,2 \ldots 9$. (Inset) Data collapse in the inner solution, with $z=\eta / \kappa^{1 / 3}$. (Right, with the same data) Rescaled phase plane focusing on the inner solution.

with $\bar{h}_{2}$. This property of the linearized equations supports the presence of oscillations as $h$ approaches $\bar{h}_{1,2}$ starting from $\eta=0^{\mp}$, as observed in numerical solutions. This contrasts with the dominantly monotone approach to $\bar{h}_{1,2}$ from the outer solution, as described above, see (3.25); this behavior is consistent with the one dimensional stable/unstable manifolds of $\bar{h}_{1}, \bar{h}_{2}$ respectively.

The structure of the solution is further illustrated with the phase plots of PDE simulations, shown in Figures 18 and 19. In Figure 18, the inner and outer solutions are clearly visible; while $\Gamma=O(\delta)$, the outer solution lies near the invariant plane $\Gamma=0$, spiraling out of $h_{L}$, and into $h_{R}$. As $\Gamma$ changes from $O(\delta)$, and approaches $\Gamma_{\max }$ approximately linearly, the outer solution lies near the one-dimensional invariant manifolds of $\bar{h}_{1}, \bar{h}_{2}$. The inner solution of (4.8) has $\Gamma \approx \Gamma_{\max }$, and this section of the trajectory is nearly horizontal in the figure.

In Figure 19, where the calculations are performed with small $\delta$ and a sequence of values of $\kappa$, the scaling of the inner solution is demonstrated. In the left hand plot, the entire graph of $h$ is shown for each $\kappa$. In the inset, and on the right hand plot, the profiles collapse under the scaling to show the inner solution, which to leading order is independent of $\kappa$. A consequence of this scaling is that the capillary ridge, corresponding to the global maximum of $h$, persists in the limit $\kappa \rightarrow 0$, while the width of the ridge scales as $\kappa^{1 / 3}$. Correspondingly, in the left plot, the maximum of $h$ (where of course $h^{\prime}=0$ ) is the same for each of the trajectories.

\section{Discussion}

In this paper, our focus has been the effects of regularizing terms on the weak traveling wave solution shown in Figure 1. We have identified an array of different behaviors depending on whether the regularizing terms are second order (with parameters $\beta, \delta$ ) or fourth order (with parameter $\kappa$ ). The interplay between the three parameters is somewhat subtle, and we have restricted attention to the cases $\kappa=0$, in Section 3 , and $\beta=0$, in Section 4.

In the second-order system of Section 3 , with $\beta>0, \delta>0$, but $\kappa=0$, we prove the existence of a one-parameter family of traveling waves, parametrized by the maximum 

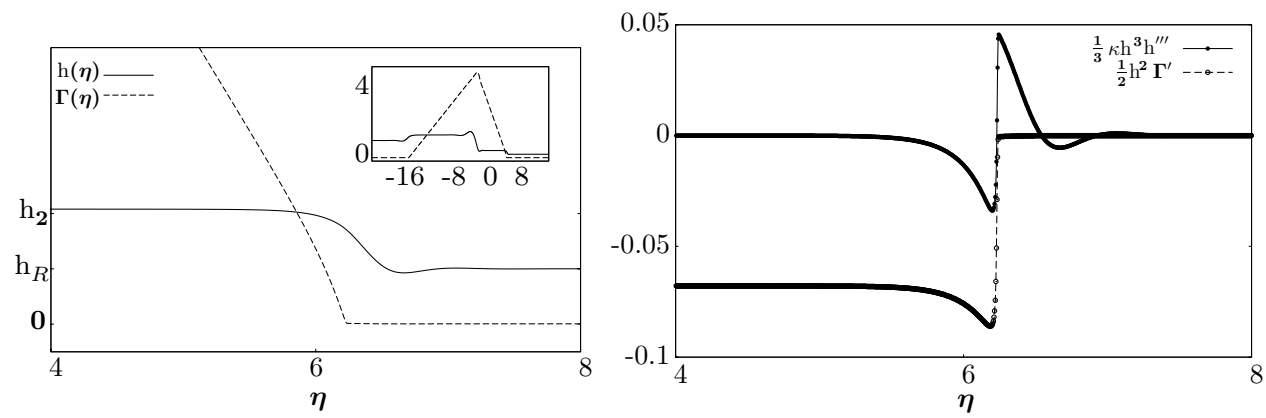

Figure 20. (Left) Graphs of $h, \Gamma$ at the leading edge of the surfactant profile. (Inset) The entire $(h, \Gamma)$ wave. (Right, with the same data) Graphs of $\frac{1}{3} \kappa h^{3} h^{\prime \prime \prime}, \frac{1}{2} h^{2} \Gamma^{\prime}$ showing the jumps at the leading edge of the surfactant.

surfactant concentration $\Gamma_{\max }$, as with the weak traveling wave. We find that as $\beta \rightarrow 0$, the solution exhibits jumps in the height $h$ and surfactant concentration gradient $\Gamma^{\prime}$ at $\eta=0$, where $\Gamma=\Gamma_{\max }$. In contrast, as $\delta \rightarrow 0$, the solution remains continuous, and is smooth at $\Gamma_{\max }$, but develops corners in $h$ and $\Gamma$ at $\eta=\eta_{1}, \eta_{2}$, which define the edges of the support of $\Gamma(\eta)$ for $\delta=0$.

When fourth-order surface tension effects dominate (with $\kappa>0, \beta=0$ in Section 4), oscillations occur in parts of the traveling wave. We observe these in numerical simulations, and provide some analysis of the overall structure in terms of asymptotics, phase portraits, and dynamical systems. As in Section 3.4, the limit $\delta \rightarrow 0$ is of great interest for the fourth-order problem. With $\delta=0, \Gamma(\eta)$ has compact support and the ODE system becomes discontinuous at $\Gamma=0$. We can write the limiting system, as we did for (3.54),

$$
\beta \frac{d h}{d \eta}-\kappa \frac{d^{3} h}{d \eta^{3}}=\left\{\begin{array}{ll}
P_{1}(h) / h^{3}, & \Gamma>0, \\
P_{0}(h) / h^{3}, & \Gamma=0,
\end{array} \quad \frac{d \Gamma}{d \eta}= \begin{cases}2 s\left(h-h_{*}\right) / h^{2}, & \Gamma>0, \\
0, & \Gamma=0 .\end{cases}\right.
$$

Consequently, the equations for $\Gamma$ and $h$ are coupled only through the switch from $\Gamma=0$ to $\Gamma>0$, and the vector field jumps at such points, corresponding to jumps in $\Gamma^{\prime}$ and either $h^{\prime}$ (if $\kappa=0$ ) or $h^{\prime \prime \prime}$ (if $\kappa>0$ ).

Jumps suggested by the discontinuous vector field in (5.1) are clearly visible in numerical simulations. In Figure 20 (left), we show the result of a finite difference numerical simulation of the solution to the PDE system (1.4ab) with $\beta=0$ and $\kappa>0$, when it has effectively converged to the traveling wave. In Figure 20(right), the computed derivatives $h^{\prime \prime \prime}(\eta)$ and $\Gamma^{\prime}(\eta)$ are shown to experience jumps at the leading edge of the surfactant distribution (i.e., where $\Gamma \rightarrow 0$ ). The magnitudes of these numerical jumps show good agreement with the predictions from the jump conditions derivable from (5.1), and more directly from $(1.4 a)$,

$$
\left[\frac{1}{3} \kappa h^{3} h^{\prime \prime \prime}\right]=\left[\frac{1}{2} h^{2} \Gamma^{\prime}\right]
$$

Finally, we close by noting that when $\delta>0$ we can re-write system (4.1 $a b)$ analogously to the framework from Section 3.5 using the scaling

$$
\Gamma=\delta \hat{\Gamma}(z), \quad h=\hat{h}(z), \quad \eta=\delta z,
$$


leading to the system of ODEs

$$
\rho \frac{d \hat{h}}{d z}-\tau \frac{d^{3} \hat{h}}{d z^{3}}=\frac{P_{1}(\hat{h}) \hat{\Gamma} \hat{h}+4 P_{0}(\hat{h})}{\hat{h}^{3}(\hat{\Gamma} \hat{h}+4)}, \quad \frac{d \hat{\Gamma}}{d z}=\frac{2 s \hat{\Gamma}\left(\hat{h}-h_{*}\right)}{\hat{h}(\hat{\Gamma} \hat{h}+4)},
$$

with parameters

$$
\rho=\frac{\beta}{\delta}, \quad \tau=\frac{\kappa}{\delta^{3}},
$$

and initial conditions $\hat{h}(0)=h_{*}, \hat{\Gamma}(0)=v^{-1}\left(v=\delta / \Gamma_{\max }\right)$. In this context, the analysis of Section 3 explored the dependence of the solutions on $(\rho, v)$ for $\tau=0$, while Section 4 is focused on the case $\tau>0$ and $\rho=0$. It is reasonable to suppose that between these limiting cases the behavior of the solutions would be similar to the behavior found in the corresponding section of the paper, and indeed numerical experiments suggest this to be the case. We conjecture that there is a threshold curve separating two types of behavior: solutions with a capillary ridge, in which $h(\eta)$ rises above $h=h_{1}$, and solutions with no capillary ridge, in which solutions are bounded above by $h_{1}$. Such a threshold is observed in the surfactant-free case studied by Bertozzi and Brenner [3]. It will be of interest to identify a corresponding threshold for the surfactant-laden traveling waves of this paper.

\section{Acknowledgments}

We acknowledge very helpful conversations with John King, and with members of the NCSU/UNC Dynamical Systems Working Group, including Anna Ghazaryan, Chris Jones, Xiao-Biao Lin, Vahag Manukian, and Steve Schecter. We would also like to acknowledge the helpful suggestions of the reviewer.

This research is supported by NSF Grants: DMS-0239125, DMS-0244498 (RL); DMS0244491, DMS-0604047 (MS); DMS-0239125 CAREER, DMS-0244498 FRG(TPW).

\section{References}

[1] Beretta, E. Hulshof, J. \& Peletier, L. A. (1996) On an ODE from forced coating flow. J. Diff. Eq., 130(1), 247-265.

[2] Bernis, F. \& Peletier, L. A. (1996) Two problems from draining flows involving third-order ordinary differential equations. SIAM J. Math. Anal., 27(2), 515-527.

[3] Bertozzi, A. L. \& Brenner, M. P. (1997) Linear stability and transient growth in driven contact lines. Phys. Fluids, 9(3):530-539.

[4] Bertozzi, A. L., Münch, A. \& Shearer, M. (1999) Undercompressive shocks in thin film flows. Physica D, 134(4), 431-464.

[5] Bowen, M., Sur, J., Bertozzi, A. L. \& Behringer, R. P. (2005) Nonlinear dynamics of two-dimensional undercompressive shocks. Physica D, 209(1-4), 36-48.

[6] Edmonstone, B. D., Matar, O. K. \& Craster, R. V. (2004) Flow of surfactant-laden thin films down an inclined plane. J. Eng. Math., 50(2-3), 141-156.

[7] Edmonstone, B. D., Matar, O. K. \& Craster, R. V. (2005) Surfactant-induced fingering phenomena in thin film flow down an inclined plane. Phys. D, 209(1-4), 62-79.

[8] Galaktionov, V. A. (2006) On higher-order viscosity approximations of odd-order nonlinear PDEs. Preprint. 
[9] Huppert, H. E. (1982) Flow and instability of a viscous current down a slope. Nature, 300, $427-429$.

[10] Jensen, O. E. \& Grotberg, J. B. (1992) Insoluble surfactant spreading on a thin viscous film: shock evolution and film rupture. J. Fluid Mech., 240, 259-288.

[11] Jensen, O. E. \& Grotberg, J. B. (1993) The spreading of heat or soluble surfactant along a thin liquid film. Phys. Fluids A, 5(1), 58-68.

[12] King, J. R. (1988) Approximate solutions to a nonlinear diffusion equation. J. Eng. Math., 22(1), 53-72.

[13] King, J. R. \& Please, C. P. (1986) Diffusion of dopant in crystalline silicon: an asymptotic analysis. IMA J. Appl. Math., 37(3), 185-197.

[14] Levy, R. \& Shearer, M. (2006) The motion of a thin film driven by surfactant and gravity. SIAM J. Appl. Maths, 66(5), 1588-1609.

[15] Dal Passo, R., Bertsch, M. \& Nitsch, C. (2005) A system of degenerate parabolic nonlinear pde's: a new free boundary problem. Interfaces Free Bound., 7, 255-276.

[16] Marangoni, C. (1865) On the expansion of a drop of liquid floating in the surface of another liquid. $\mathrm{PhD}$ thesis, University of Pavia.

[17] Matar, O. K. (2002) Nonlinear evolution of thin free viscous films in the presence of soluble surfactant. Phys. Fluids, 14, 4216-4234.

[18] Мock, M. S. (1976) On fourth-order dissipation and single conservation laws. Commun. Pure Appl. Math., 29, 383-388.

[19] Moriarty, J. A., Schwartz, L. W. \& Tuck, E. O. (1991) Unsteady spreading of thin liquid films with small surface tension. Phys. Fluids A, 3(5), 733-742.

[20] MüNCH, A. (2003) Pinch-off transition in Marangoni-driven thin films. Phys. Rev. Lett., 91(1), 016105.

[21] MüNCH, A. \& Wagner, B. A. (1999) Numerical and asymptotic results on the linear stability of a thin film spreading down a slope of small inclination. Eur. J. Appl. Math., 10(3), 297-318.

[22] Myers, T. G. (1998) Thin films with high surface tension. SIAM Rev., 40(3), 441-462.

[23] Oron, A., Davis, S. H. \& Bankoff, S. G. (1997) Long-scale evolution of thin liquid films. Rev. Modern Phys., 69(3), 931-980.

[24] Renardy, M. (1996) A singularly perturbed problem related to surfactant spreading on thin films. Nonlinear Anal., 27(3), 287-296.

[25] Renardy, M. (1997) A degenerate parabolic-hyperbolic system modeling the spreading of surfactants. SIAM J. Math. Anal., 28(5), 1048-1063.

[26] Schaeffer, D. G. \& Shearer, M. (1987) Riemann problems for nonstrictly hyperbolic $2 \times 2$ systems of conservation laws. Trans. AMS, 301, 267-306.

[27] Stone, H. A. (1990) A simple derivation of the time-dependent convective-diffusion equation for surfactant transport along a deforming interface. Phys. Fluids A, 2(1), 111-112.

[28] Troy, W. C. (1993) Solutions of third-order differential equations relevant to draining and coating flows. SIAM J. Math. Anal., 24(1), 155-171.

[29] Tuck, E. O. \& Schwartz, L. W. (1990) A numerical and asymptotic study of some thirdorder ordinary differential equations relevant to draining and coating flows. SIAM Rev., 32, 453-469.

[30] Warner, M. R. E. \& Craster, R.V. (2004) Fingering phenomena created by a soluble surfactant deposition on a thin liquid film. Phys. Fluids, 16, 2933-2951.

[31] Witelski, T. P., Shearer, M. \& Levy, R. (2006) Growing surfactant waves in thin liquid films driven by gravity. Appl. Math. Res. Exp., 2006(15487), 1-21. 